\title{
An Experimental Analysis of Multidimensional Cheap Talk*
}

\author{
Ernest K. Lai ${ }^{\dagger}$ \\ Wooyoung $\operatorname{Lim}^{\ddagger}$ \\ Joseph Tao-yi Wang§
}

April 8, 2015

\begin{abstract}
We design experimental games that capture the logic of Battaglini's (2002) construction of fully revealing equilibrium in multidimensional cheap talk. Two senders transmit information to a receiver over a $2 \times 2$ state space. Despite overall misaligned interests, full revelation is achieved in equilibrium by having the senders truthfully reveal along distinct dimensions. Our experimental findings confirm that more information can be extracted with two senders in a multidimensional setting. The extent to which information is transmitted depends on whether dimensional interests are aligned between a sender and the receiver, the sizes of the message spaces, and the specification of out-of-equilibrium beliefs. While inducing interest alignment on the relevant dimensions and restricting the message spaces facilitated equilibrium play and information transmission, having a fully revealing equilibrium that is supported by implausible beliefs reduced the instances in which the equilibrium was played.

\section{Keywords: Strategic Information Transmission; Multidimensional Cheap Talk; Fully Revealing Equilibrium; Robust Equilibrium; Laboratory Experiment}

JEL classification: C72; C92; D82; D83

\footnotetext{
*We are grateful to Attila Ambrus, Marco Battaglini, Colin Camerer, Colin Campbell, Vincent Crawford, Shachar Kariv, Navin Kartik, Alec Smith, Joel Sobel and Satoru Takahashi for their valuable comments and suggestions. We are especially grateful to the anonymous advisory editor and referees, whose comments and suggestions helped significantly improve the paper. We thank the conference and seminar participants at the 2011 International ESA Conference, the 22nd International Conference on Game Theory, the 2011 Asian Meeting of the Econometric Society, the Fall 2011 Midwest Economic Theory Conference, the 2011 North-American ESA Conference, the 3rd Annual Xiamen University International Workshop on Experimental Economics and Finance, the 2013 North American Summer Meeting of the Econometric Society, Korea Institute of Public Finance, Nanyang Technological University, The Chinese University of Hong Kong, Peking University HSBC School of Business, Rutgers University, The Hong Kong University of Science and Technology, California State University Long Beach (Mathematics Department), Korea University, National University of Singapore, Singapore Management University, Sogang University, and Seoul National University for their helpful comments and discussion. We gratefully acknowledge the financial support (grant no.: NSC99-2410-H-002-060-MY3) from the National Science Council of Taiwan. This paper was previously circulated and presented under the title "Experimental Implementations and Robustness of Fully Revealing Equilibria in Multidimensional Cheap Talk."

${ }^{\dagger}$ Department of Economics, Lehigh University. Email: kwl409@lehigh.edu.

${ }^{\ddagger}$ Department of Economics, The Hong Kong University of Science and Technology. Email: wooyoung@ust.hk.

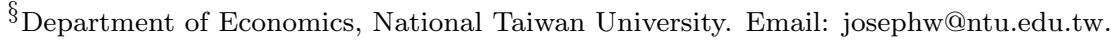




\section{Introduction}

A defining hallmark of modern economies is the extensive specialization that occurs not only in physical production but also in the more intangible domain of decision making and information provision. Comparative advantage not only dictates that decision makers delegate knowledge acquisition to experts but also guides different experts to specialize in offering advice on distinct issues. When conflicts of interests are present, strategic considerations may provide yet another reason for decision makers to consult different experts. In seeking advice from an interested advisor on the potential impacts of a bill, a legislator may obtain impartial advice only in certain areas, creating the need for her to consult another advisor who might be forthright in a different manner. In a seminal paper on multidimensional cheap talk, Battaglini (2002) provides a strategic argument for otherwise equally informed experts to specialize in giving advice on different dimensions. ${ }^{1}$

The theory of multidimensional cheap talk contrasts sharply with its unidimensional counterpart. In the canonical cheap-talk model of Crawford and Sobel (1982), unless interests are perfectly aligned between the sender and the receiver, only partial information can be transmitted. ${ }^{2}$ The picture changes drastically when one more sender is introduced and the uncertainty becomes multidimensional. Battaglini (2002) shows that with two senders and for a multidimensional (unbounded) state space, a fully revealing equilibrium exists even when the senders are arbitrarily biased. The key insight of the equilibrium construction is that, by having one sender truthfully reveal along one dimension, an incentive is created for the other sender to truthfully reveal along the other dimension. By combining the information from the two senders, the receiver fully identifies the state. ${ }^{3}$

We experimentally investigate the key insight of Battaglini's (2002) construction. In particular, we address three research questions. First, we explore under what circumstances the theoretical prediction that more information can be extracted with two senders in a mul-

\footnotetext{
${ }^{1}$ Cheap-talk models have been a theoretical arena for studying the strategic interactions between experts and decision makers. Other than the interactions between legislators and advisors (Gilligan and Krehbiel, 1989; Krishna and Morgan, 2001b), they have shed light on, for example, the interactions between stock analysts and investors (Morgan and Stocken, 2003) and between doctors and patients (Köszegi, 2006).

${ }^{2}$ This informational property of the equilibrium survives modeling variations within the single-sendersingle-dimension environment. It is invariant to, for example, the introduction of an additional round of communication (Krishna and Morgan, 2004), noise in the communication channel (Blume et al., 2007), and a mediator (Goltsman et al., 2009; Ivanov, 2010).

${ }^{3}$ For an analysis of multiple senders with unidimensional or discrete state spaces, see Gilligan and Krehbiel (1989), Austen-Smith (1993), and Krishna and Morgan (2001a,b). Battaglini (2002) revisits the problem with more complete characterizations. Ambrus and $\mathrm{Lu}$ (2014) and $\mathrm{Lu}$ (2015) further investigate robust equilibria in such an environment. For an analysis of multidimensional state spaces with a single sender, see Levy and Razin (2007) and Chakraborty and Harbaugh (2007, 2010). For papers that introduce additional receivers, see Farrell and Gibbons (1989) and Goltsman and Pavlov (2011).
} 
tidimensional setting will be verified in the lab, and whether the information transmission will be conducted in strategies consistent with the logic of Battaglini's (2002) equilibrium. Second, we investigate how, in an experimental setting, the size of the message spaces may affect the extent of information transmission. Finally, we explore how the empirical performance of an equilibrium may depend on the specification of out-of-equilibrium beliefs, which, unlike the case with one sender, is an important issue in cheap talk with multiple senders. ${ }^{4}$

We create simple discrete environments to address our research questions. Two senders, Sender 1 (he) and Sender 2 (he), send simultaneous messages to a receiver (she) regarding a 2 (horizontal dimension) $\times 2$ (vertical dimension) state space. The receiver chooses among four actions. Each sender has available four costless messages framed as non-binding action recommendations. Interests are overall misaligned: the players' ideal actions differ. However, each sender and the receiver share a common ranking of the relevant actions assuming that the senders' influences on the receiver are limited to distinct dimensions. Such a payoff structure supports a fully revealing equilibrium in which Sender 1 truthfully reveals along the horizontal dimension and Sender 2 along the vertical dimension.

We consider treatment variations in which the message spaces become binary and in which the common interest between a sender and the receiver is along a diagonal of the state space matrix. One-sender control games are also included. To address the issue of out-of-equilibrium beliefs, we design a game by eliminating a state so that only three states remain. Following Ambrus and Takahashi (2008), out-of-equilibrium messages arise in the game as messages that indicate a "state" outside of the state space, i.e., the eliminated state. The corresponding fully revealing equilibrium is supported by out-of-equilibrium beliefs that are implausible according to the robustness criterion advanced by Battaglini $(2002) .^{5}$

\footnotetext{
${ }^{4}$ When there is only one sender, out-of-equilibrium messages arise only as unused messages, which can be disregarded without any impact on equilibrium outcomes. With two senders, out-of-equilibrium messages can arise when they convey inconsistent information. Even when the senders reveal along different dimensions, as in Battaglini's (2002) construction, so that there will be no inconsistent information, Ambrus and Takahashi (2008) point out that out-of-equilibrium messages can still emerge: if the state space is bounded, after a deviation the messages may indicate a "state" outside of the state space. (Intuitively, when one investment advisor advocates strongly for stocks and another strongly for bonds, investors are likely to raise question if there does not exist economic condition that warrants heavy investments in both.) Accordingly, the specification of out-of-equilibrium beliefs is an indispensable part of the characterizations of cheap-talk equilibria with multiple senders, which further leads to the robustness inquiry of what equilibria can only be supported by implausible beliefs. While "implausibility" in this regard is based on theoretical consideration, an experimental study that investigates this issue may inform the theory by exploring whether the theoretical notion has empirical appeal supported by layman's intuition.

${ }^{5}$ For a few findings on robustness in the theoretical literature, see Battaglini (2002) who shows that the fully revealing equilibrium under an unbounded state space is robust to noise in the senders' obser-
} 
Our simple discrete design provides an environment that is relatively easy for subjects to comprehend. To take advantage of such a simple design, however, we need to depart from certain aspects of Battaglini's (2002) model. In particular, in his model a dimension of common interest between a sender and the receiver is endogenous (i.e., it is an equilibrium phenomenon), whereas in our case it arises from the payoff structure of the game. Despite this departure from Battaglini (2002), we nevertheless preserve the key logic of his equilibrium in which one sender reveals along one dimension of common interest.

In our findings from the four-message games, high adherence to fully revealing equilibrium was observed when a sender's and the receiver's interests are aligned on a dimension regardless of the other sender's behavior. Sender 1s revealed on the horizontal dimension and randomized on the vertical dimension, and vice versa for Sender 2s. Receivers filtered information accordingly, following the senders' recommendations selectively on the separate dimensions they revealed. When the alignment of interests in some states depends on the other sender's behavior, lower adherence was observed. Nevertheless, receivers in general identified true states more often with two senders than with one sender.

Findings from binary-message games showed drastically higher adherence. With each sender constrained to recommending only one dimension, which eliminated the occurrence of inconsistent messages, receivers virtually always followed the recommendations. Senders in turn followed the prescriptions of fully revealing equilibrium significantly more often, notably in the games in which low adherence was observed in the four-message counterparts. Lower adherence was again observed under a restricted state space with three states. Furthermore, after messages indicating that a deviation had occurred, receivers took the responses that in theory do not support the equilibrium.

Our findings provide answers to our three research questions. Even though the games all admit fully revealing equilibria, the observed extent of information transmission depends on the nature of dimensional common interests that is embodied in the payoff structure of the game. This result contrasts with Battaglini's (2002) theory in which a dimension of common interest is endogenous. Yet our findings confirm that extracting more information via the strategies in which the senders reveal along different dimensions is implementable in the lab. This is especially the case when the message spaces are restricted to coincide with the equilibrium dimension of each sender. Our findings thus highlight the role of message spaces in facilitating information transmission in a multidimensional setting. Regarding

vations; under a different information structure, Levy and Razin (2007) show that it is not; Ambrus and Takahashi (2008) show that imposing the so-called "diagonal continuity" drastically reduces the possibility of full revelation under a bounded state space; Kim (2010) proposes yet another criterion - "outcomerobustness" - and shows that no fully revealing equilibrium studied in Levy and Razin (2007) survives. 
the specification of out-of-equilibrium beliefs, we demonstrate that theoretically robust equilibria are also empirically plausible: they are more likely to be implemented in the lab than are equilibria that need to be supported by implausible beliefs.

Until recently, the experimental literature on communication games has focused on one sender and one receiver. Examples are Dickhaut et al. (1995), Blume, et al. (1998, 2001), Gneezy (2005), Cai and Wang (2006), Sánchez-Pagés and Vorsatz (2007, 2009), Kawagoe and Takizawa (2009), and Wang et al. (2010). ${ }^{6}$ As the first experimental study that departs from this trend on the receiver's side, Battaglini and Makarov (2014) design an experiment that tests the prediction of Farrell and Gibbons (1989). On the sender's side, a political science study by Minozzi and Woon (2015) also examines, similar to what we do, games with two senders, but their design features a single-dimension environment in which the senders' biases are private information.

Another experimental study on multidimensional cheap talk that is also motivated by Battaglini (2002) is Vespa and Wilson (2014). Their independent work uses circles to represent the multidimensional state space. In terms of the complementarity of our designs, their larger state space complements our simple one by considering a richer environment; our design complements theirs by serving as a simple benchmark to identify whether the extra complexity in their design compared to ours may contribute to different observed behavior. In terms of a major characteristic difference, they adopt the circular design partly to avoid the consideration of out-of-equilibrium beliefs, whereas an important contribution of our design is to allow us to empirically assess the impacts of out-of-equilibrium belief specification, which, as mentioned above, is a fundamental issue in the theoretical literature on multi-sender cheap talk. With respect to findings, they observe that while full information revelation could be implemented in the lab, partial revelation was the norm; senders followed exaggeration strategies while receivers adopted different heuristics, and Vespa and Wilson (2014) suggest the possibility of using level- $k$ reasoning and analogy-based expectation to rationalize the observed behavior. ${ }^{7}$ Our finding that the fully revealing equilibria could be implemented under certain game environments complements some of these findings. On the other hand, our findings on the comparative statics between one-sender games and two-sender games, the effect of the sizes of the message spaces, and the effect of the robustness of the equilibria in question represent our unique contributions.

\footnotetext{
${ }^{6}$ See also Crawford (1998) for a survey of earlier studies.

${ }^{7}$ See Crawford (2003) for a pioneering theoretical work on applying level- $k$ reasoning to communication games. The analogy-based expectation equilibrium is developed by Jehiel (2005), and Jehiel and Koessler (2008) apply the alternative equilibrium concept to analyze communication in Crawford and Sobel's (1982) model.
} 
The exposition of the rest of this paper is as follows. Section 2 presents the theoretical framework that guides the design of our experimental games. Section 3 introduces the games and derives the equilibrium predictions. Section 4 formulates the experimental hypotheses and describes the experimental procedures. Section 5 reports the findings. Section 6 concludes.

\section{Two-Dimensional Cheap-Talk Games: The Theo- retical Framework}

The design of our experimental games is guided by the following theoretical framework. Uncertainty is represented by a discrete state of the world with two dimensional components, each being a binary variable: $(H, V) \in\{L, R\} \times\{U, D\}$. The common prior is that the four states are equally likely. The players are a receiver and one or two senders.

In the two-sender games, after observing the state, Sender $i, i=1,2$, sends a cheaptalk message, $m \in M_{i}$, to the receiver. ${ }^{8}$ Messages are sent simultaneously, after which the receiver takes an action $a \in A=\left\{a_{L U}, a_{R U}, a_{L D}, a_{R D}\right\}$. A behavioral strategy of Sender $i$ is $\sigma_{i}:\{L, R\} \times\{U, D\} \rightarrow \Delta M_{i}$ and that of the receiver is $\rho: M_{1} \times M_{2} \rightarrow \Delta A$. The receiver's belief function is $\mu: M_{1} \times M_{2} \rightarrow \Delta(\{L, R\} \times\{U, D\})$. Payoffs are determined by state and action. The solution concept is perfect Bayesian equilibrium, where strategies are optimal given beliefs and beliefs are derived from Bayes' rule whenever possible.

In Battaglini's (2002) fully revealing equilibrium, one sender revealing along one dimension creates an incentive for the other sender to reveal along the other dimension. The majority of our two-sender games have the following preference property, which allows us to capture this key logic of Battaglini's (2002) equilibrium construction:

1. Sender 1 and the receiver:

(a) Fixing $V=U$, both prefer $a_{L U}$ to $a_{R U}$ when $H=L$ and $a_{R U}$ to $a_{L U}$ when $H=R$.

(b) Fixing $V=D$, both prefer $a_{L D}$ to $a_{R D}$ when $H=L$ and $a_{R D}$ to $a_{L D}$ when $H=R$.

\footnotetext{
${ }^{8}$ Theoretically, the size of the message spaces has no significance as long as it does not constrain the set of equilibrium outcomes. This will be the case, for example, for binary and quadruple message spaces, which will be used in our experimental design.
} 
2. Sender 2 and the receiver:

(a) Fixing $H=L$, both prefer $a_{L U}$ to $a_{L D}$ when $V=U$ and $a_{L D}$ to $a_{L U}$ when $V=D$.

(b) Fixing $H=R$, both prefer $a_{R U}$ to $a_{R D}$ when $V=U$ and $a_{R D}$ to $a_{R U}$ when $V=D$.

To illustrate how this preference property can be exploited for full revelation, suppose that $(L, D)$ is realized and Sender 1 truthfully reveals (only) that $H=L$ (and the receiver believes him). This forces Sender 2 to choose between $a_{L U}$ and $a_{L D}$, the respective actions that the receiver will take when she believes that the state is most likely $(L, U)$ and $(L, D)$. Because Sender 2 prefers $a_{L D}$ to $a_{L U}$ in state $(L, D)$, he will prefer to tell that $V=D$. Given that Sender 2 truthfully reveals that $V=D$, Sender 1 will also, by a similar argument, prefer to tell that $H=L$. The true state $(L, D)$ is thus revealed to the receiver. The same argument applies to the other states.

In Battaglini's (2002) model, a dimension of common interest between a sender and the receiver is endogenous (i.e., it is an equilibrium phenomenon). Within the confines of a simple design, we depart from this feature in that each sender has a designated dimension of common interest with the receiver, in most cases horizontal (dimension $H$ ) for Sender 1 and vertical (dimension $V$ ) for Sender 2.

Table 1: An Example of Preference Orders over State-Action: Sender 1 Revealing on Dimension $H$ and Sender 2 on Dimension $V$ Constitute an Equilibrium

\begin{tabular}{|c|c|c|c|c|}
\cline { 2 - 5 } \multicolumn{1}{c|}{} & $(L, U)$ & $(R, U)$ & $(L, D)$ & $(R, D)$ \\
\hline Sender 1 & $a_{L D}>a_{L U}>a_{R D}>a_{R U}$ & $a_{R D}>a_{R U}>a_{L D}>a_{L U}$ & $a_{R U}>a_{L D}>a_{L U}>a_{R D}$ & $a_{R U}>a_{R D}>a_{L U}>a_{L D}$ \\
\hline Sender 2 & $a_{R U}>a_{L U}>a_{R D}>a_{L D}$ & $a_{L D}>a_{R U}>a_{L U}>a_{R D}$ & $a_{R D}>a_{L D}>a_{R U}>a_{L U}$ & $a_{L D}>a_{R D}>a_{L U}>a_{R U}$ \\
\hline Receiver & $a_{L U}>a_{R U}>a_{L D}>a_{R D}$ & $a_{R U}>a_{L U}>a_{R D}>a_{L D}$ & $a_{L D}>a_{L U}>a_{R D}>a_{R U}$ & $a_{R D}>a_{R U}>a_{L D}>a_{L U}$ \\
\hline
\end{tabular}

Table 1 presents an example of preference orders that satisfy the above preference property and thus support a fully revealing equilibrium. ${ }^{9}$ Note that interests are overall misaligned (i.e., the ideal actions do not coincide): in state $(H, V)$, the ideal action of the receiver is $a_{H V}$, but $a_{H V}$ is the second most preferred action of the senders. Despite

\footnotetext{
${ }^{9}$ There is more than one set of preference orders that satisfy the property. Two of our experimental games (to be introduced in Section 3) are direct parameter specifications of the preference orders presented in Table 1. Appendix A contains other sets of preference orders on which our other games are based.
} 
our pre-determined dimensions of common interests, we thus preserve the key theoretical prediction of Battaglini (2002) that in an environment with otherwise misaligned interests, full revelation can be achieved by having the senders reveal along different dimensions. ${ }^{10}$

\section{Experimental Design and Equilibrium Predictions}

\subsection{Five Games with Two-Dimensional Messages}

Table 2: Eight Experimental Games

\begin{tabular}{lcccccc}
\hline \hline $\begin{array}{l}\text { Game/ } \\
\text { Treatment }\end{array}$ & $\begin{array}{l}\text { No. of } \\
\text { Senders }\end{array}$ & $\begin{array}{c}\text { Messages } \\
\text { per Sender }\end{array}$ & $\begin{array}{c}\text { No. of } \\
\text { States }\end{array}$ & $\begin{array}{c}\text { All-State } \\
\text { Dim. Align. }\end{array}$ & $\begin{array}{c}\text { Out-of-Eq. } \\
\text { Messages }\end{array}$ & $\begin{array}{l}\text { Fully Revealing Equilibrium } \\
\text { (Dimensions) [Robust] }\end{array}$ \\
\hline 1 & 1 & 4 & 4 & No & $0-2$ & No \\
1-DAL & 1 & 4 & 4 & Yes & $0-2$ & No \\
\hline 2 & 2 & 4 & 4 & No & $0-12$ & Yes $(H ; V)$ [Some] \\
2 -DAL & 2 & 4 & 4 & Yes & $0-12$ & Yes (Multiple) [Some] \\
2-LAB & 2 & 4 & 4 & No & $0-12$ & Yes (Diagonal; $V)[$ Some] \\
\hline 2-2/M & 2 & 2 & 4 & No & 0 & Yes $(H ; V)[$ All $]$ \\
2-LAB-2/M & 2 & 2 & 4 & No & 0 & Yes $($ Diagonal; $V)[$ All] \\
\hline 2-2/M-3/S & 2 & 2 & 3 & No & 1 & Yes $(H ; V)[$ None] \\
\hline \hline
\end{tabular}

Note: "All-State Dim. Align." refers to whether each sender's and the receiver's ideal actions share a common dimensional component in all states. "Out-of-Equilibrium Messages" refers to the possible number of out-of-equilibrium messages per sender in any most informative equilibrium. "Dimensions" refers to the equilibrium-relevant dimensions (Sender 1; Sender 2); "Multiple" means that revealing the diagonals and revealing along dimension $H / V$ by a sender are both consistent with equilibrium. "Robust" refers to whether the fully revealing equilibria are robust according to Definition 1 below. See also Figure 11 in Appendix C for a summary of our experimental design.

Table 2 provides an overview of our eight experimental games. This subsection covers five games (two one-sender games and three two-sender games) that have two-dimensional messages, where a sender's message space contains four elements. In translating the theoretical framework into experimental games, we label action $a_{L U}$ by (left, up), $a_{R U}$ by (right, up), $a_{L D}$ by (left, down), and $a_{R D}$ by (right, down). From now on, we use $(h, v)$ to denote a generic action. We frame the information transmission problem as the sender(s) providing

\footnotetext{
${ }^{10}$ Our design is shaped by two considerations: to create an environment as simple as possible that is conducive to subjects' comprehension of the problem (Binmore, 1999) and to capture the key insight of Battaglini's (2002) equilibrium construction. The simplification necessarily entails discrepancies with Battaglini (2002). In addition to the issue of endogenous dimensions of common interests, for example, the term "dimension" also carries different meanings: in Battaglini (2002), it refers to the dimension of a vector space (the two-dimensional Euclidean state space), while we use the term to refer to the components of our discrete state.
} 
action recommendations and assign literal meaning to the messages accordingly; Sender $i$ 's message space is: $M_{i}=\{$ "(h,v)"|"(left, up)", "(right, up)", "(left, down)", "(right, down)" $\} .{ }^{11}$

Figure 1 depicts the payoff profiles of the two-sender games. Each game is represented by four tables, each table corresponds to a state, and each cell in a table contains the payoffs of Sender 1, Sender 2 and the receiver when an action is taken in the state.

\begin{tabular}{|c|ccc|ccc|}
\hline \multicolumn{1}{|c|}{ State: $(L, U)$} \\
\hline Action & \multicolumn{3}{|c|}{ left } & \multicolumn{4}{c|}{ right } \\
\hline up & 20 & 20 & 50 & 0 & 50 & 20 \\
\hline down & 50 & 0 & 10 & 10 & 10 & 0 \\
\hline
\end{tabular}

\begin{tabular}{|c|cccc|ccc|}
\hline \multicolumn{7}{|c|}{ State: $(R, U)$} \\
\hline Action & \multicolumn{3}{|c|}{ left } & \multicolumn{4}{c|}{ right } \\
\hline up & 0 & 15 & 20 & 20 & 20 & 50 \\
\hline down & 10 & 60 & 0 & 50 & 0 & 10 \\
\hline
\end{tabular}

\begin{tabular}{|c|ccc|ccc|}
\hline \multicolumn{1}{|c|}{ State: $(L, D)$} \\
\hline Action & \multicolumn{3}{|c|}{ left } & \multicolumn{4}{c|}{ right } \\
\hline up & 15 & 0 & 20 & 60 & 10 & 0 \\
\hline down & 20 & 20 & 50 & 0 & 50 & 10 \\
\hline
\end{tabular}

\begin{tabular}{|c|ccc|ccc|}
\hline \multicolumn{8}{|c|}{ State: $(R, D)$} \\
\hline Action & \multicolumn{3}{|c|}{ left } & \multicolumn{4}{c|}{ right } \\
\hline up & 10 & 10 & 0 & 50 & 0 & 20 \\
\hline down & 0 & 50 & 10 & 20 & 20 & 50 \\
\hline
\end{tabular}

(a) Game $2(2-2 / \mathrm{M})$

\begin{tabular}{|c|ccc|ccc|}
\hline \multicolumn{1}{|c|}{ State: $(L, U)$} \\
\hline Action & \multicolumn{3}{|c|}{ left } & \multicolumn{3}{c|}{ right } \\
\hline up & 20 & 20 & 50 & 0 & 50 & 20 \\
\hline down & 10 & 10 & 0 & 50 & 0 & 10 \\
\hline
\end{tabular}

\begin{tabular}{|c|ccc|ccc|}
\hline \multicolumn{7}{|c|}{ State: $(R, U)$} \\
\hline Action & \multicolumn{3}{|c|}{ left } & \multicolumn{4}{c|}{ right } \\
\hline up & 0 & 15 & 20 & 20 & 20 & 50 \\
\hline down & 50 & 0 & 10 & 10 & 60 & 0 \\
\hline
\end{tabular}

\begin{tabular}{|c|ccc|crr|}
\hline \multicolumn{8}{|c|}{ State: $(L, D)$} \\
\hline Action & \multicolumn{3}{|c|}{ left } & \multicolumn{4}{c|}{ right } \\
\hline up & 10 & 10 & 0 & 50 & 0 & 20 \\
\hline down & 20 & 20 & 50 & 0 & 50 & 10 \\
\hline
\end{tabular}

\begin{tabular}{|c|ccc|ccc|}
\hline \multicolumn{1}{|c|}{ State: $(R, D)$} \\
\hline Action & \multicolumn{3}{|c|}{ left } & \multicolumn{4}{c|}{ right } \\
\hline up & 15 & 0 & 20 & 60 & 10 & 0 \\
\hline down & 0 & 50 & 10 & 20 & 20 & 50 \\
\hline
\end{tabular}

(b) Game 2-LAB (2-LAB-2/M)

\begin{tabular}{|c|rrl|rll|}
\hline \multicolumn{7}{|c|}{ State: $(L, U)$} \\
\hline Action & \multicolumn{3}{|c|}{ left } & \multicolumn{3}{c|}{ right } \\
\hline up & 20 & 20 & 50 & 0 & 50 & 0 \\
\hline down & 50 & 0 & 0 & 10 & 10 & 20 \\
\hline
\end{tabular}

\begin{tabular}{|c|rll|rrr|}
\hline \multicolumn{1}{|c|}{ State: $(R, U)$} \\
\hline Action & \multicolumn{3}{|c|}{ left } & \multicolumn{4}{c|}{ right } \\
\hline up & 0 & 50 & 0 & 20 & 20 & 50 \\
\hline down & 10 & 10 & 20 & 50 & 0 & 0 \\
\hline
\end{tabular}

\begin{tabular}{|c|rrl|rll|}
\hline \multicolumn{1}{|c|}{ State: $(L, D)$} \\
\hline Action & \multicolumn{3}{|c|}{ left } & \multicolumn{3}{c|}{ right } \\
\hline up & 50 & 0 & 0 & 10 & 10 & 20 \\
\hline down & 20 & 20 & 50 & 0 & 50 & 0 \\
\hline
\end{tabular}

\begin{tabular}{|c|rll|rrl|}
\hline \multicolumn{1}{|c|}{ State: $(R, D)$} \\
\hline Action & \multicolumn{3}{|c|}{ left } & \multicolumn{4}{c|}{ right } \\
\hline up & 10 & 10 & 20 & 50 & 0 & 0 \\
\hline down & 0 & 50 & 0 & 20 & 20 & 50 \\
\hline
\end{tabular}

(c) Game 2-DAL

Figure 1: Payoff Profiles of Games with Two Senders and Four States

\footnotetext{
${ }^{11}$ For expositional clarity, throughout the paper we use quotation marks to distinguish between actions and messages. No such distinction was made in the experiment.
} 
The payoffs in Game 2 represent a parameter specification of the preference orders in Table 1. The game forms the pivot of our design, where every other game is essentially one or two properties away from it. We omit Sender 2, which gives us Game 1 (not depicted). ${ }^{12}$ We interchange the payoffs under actions (left, down) and (right, down) and then further interchange the payoff profiles under states $(L, D)$ and $(R, D)$. This represents a relabeling of the states and actions in Game 2, and we call the resulting game Game 2-LAB.

To introduce the third two-sender game, consider state $(L, U)$ in Game 2 as an illustration. The receiver's ideal action is (left, up), while that of Sender 1 is (left, down) and that of Sender 2 is (right, up). Although the ideal actions differ, the receiver's ideal action shares the common dimensional component $h$ (i.e., left) with Sender 1's ideal action, and it shares the common $v$ (i.e., up) with Sender 2's ideal action. We call such a property $d i$ mensional alignment (DAL). In Game 2, dimensional alignment does not exist for Sender 1 in state $(L, D)$ or for Sender 2 in $(R, U)$. We create a game in which dimensional alignment is in place for both senders in all states and call such a game with all-state dimensional alignments Game 2-DAL. ${ }^{13}$ Given the structure of the game, a consequence of all-state dimensional alignments is that, when the receiver's ideal action is (left, up) as in state $(L, U)$, Sender 1 [Sender 2] prefers (left, $v)[(h$, up $)]$ over (right, $v)[(h$, down $)]$ for any fixed $v[h]$, and this holds for all states in Game 2-DAL. Game 1-DAL (not depicted) is derived from Game 2-DAL by omitting Sender 2.

We analyze the most informative equilibria, which are fully revealing for the two-sender games. We say that a sender truthfully reveals along dimension $H(V)$ if he reveals whether the state consists of $L$ or $R$ ( $U$ or $D)$; a sender is said to truthfully reveal the diagonals if he reveals whether the state is on the major diagonal $\{(L, U),(R, D)\}$ or on the minor $\{(R, U),(L, D)\}$. We group all fully revealing equilibria with the same information partition provided by each sender into a class; equilibria within a class thus differ only by different uses of messages to induce the unique information partition. ${ }^{14}$

Proposition 1. There exists a fully revealing equilibrium in Games 2, 2-LAB, and 2-DAL. For Games 2 and 2- $L A B$,

1. there is a class of fully revealing equilibria exclusive to Game 2, in which Sender 1

\footnotetext{
${ }^{12}$ The game-naming convention is that the number, 1 or 2 , indicates the number of sender(s) and any suffix represents a manipulation relative to Game 2 or its derivative.

${ }^{13}$ Refer to Appendix A for the sets of preference orders on which Games 2-LAB (Table 5) and 2-DAL (Table 6) are based. Note that in Game 2-LAB, the appropriately redefined "diagonal alignment" is in place for Sender 1 in all states except $(R, D)$, and for Sender 2 the dimensional alignment profile remains the same as that in Game 2.

${ }^{14}$ As usual in cheap-talk games, in our games there is an inessential multiplicity of equilibria with different uses of messages supporting a given equilibrium outcome.
} 
truthfully reveals only along dimension $H$ and Sender 2 only along dimension $V$;

2. there is a class of fully revealing equilibria exclusive to Game 2-LAB, in which Sender 1 truthfully reveals only the diagonals and Sender 2 only along dimension $V$; and,

3. there is a class of fully revealing equilibria that exists in both games, in which both Sender 1 and Sender 2 truthfully reveal all four states.

All three classes of equilibria exist in Game 2-DAL. There is an additional class of fully revealing equilibria exclusive to Game 2-DAL, in which Sender 1 truthfully reveals only along dimension $H$ and Sender 2 only the diagonals.

We call the equilibria in Proposition 1.1 dimensionally revealing equilibria and those in Proposition 1.2 diagonally revealing equilibria. The rationale behind the dimensionally revealing equilibria in Game 2, in which the equilibrium-relevant dimension is $H$ for Sender 1 and $V$ for Sender 2, follows from the discussion in Section 2. ${ }^{15}$ The relabeling in Game 2-LAB effectively interchanges states $(L, D)$ and $(R, D)$ for Sender 1's revelation in the diagonally revealing equilibria. In Game 2-DAL, under the all-state dimensional alignments and given that the receiver's ideal actions are the senders' second most preferred, the game admits not only dimensionally revealing equilibria but also diagonally revealing equilibria. ${ }^{16}$

For the dimensionally revealing equilibrium in Game 2-DAL, the all-state dimensional alignments lead to some type of "dominance." 17 Conditional on the receiver following the other sender's recommendation on his equilibrium-relevant dimension, regardless of whether it is truthful or not, a sender's interest is always aligned with the receiver's along his own dimension. Accordingly, it is "dominant" for each sender to truthfully reveal along his equilibrium-relevant dimension. This property does not hold for the diagonally revealing equilibrium; for both classes, there exists a state in which a sender strictly prefers to deviate from truthful revelation unless he believes that the other sender truthfully reveals with a probability of $\frac{2}{3}$ or above. ${ }^{18}$ Similarly, a belief of at least $\frac{9}{13}$ is required for the

\footnotetext{
${ }^{15}$ All of the proofs are relegated to Appendix B.

${ }^{16}$ The classes of fully revealing equilibria in Proposition 1 are meant to be representative but not exhaustive. There exist fully revealing equilibria with hybrid strategy profiles in which, for example, Sender 1 truthfully reveals all four states and Sender 2 only along dimension $V$.

${ }^{17}$ For expositional convenience, from now on we use "equilibrium" to mean a class of equilibria unless the context suggests otherwise.

${ }^{18}$ As an illustration, consider Sender 1 in state $(L, U)$ in the equilibrium in which he reveals only the diagonals. Suppose Sender 1 believes that Sender 2 truthfully reveals $U$ with probability $q$. Then, assuming that the receiver believes Sender 2, Sender 1's expected payoff from truthfully revealing the major diagonal $\{(L, U),(R, D)\}$ is $20 q+10(1-q)$, and that from revealing the minor diagonal $\{(R, U),(L, D)\}$ is $50(1-q)$. Accordingly, Sender 1 prefers to truthfully reveal if and only if $20 q+10(1-q) \geqslant 50(1-q)$ or $q \geqslant \frac{2}{3}$.
} 
equilibria in Games 2 and 2-LAB. In terms of less strategic uncertainty from the senders' side, the dimensionally revealing equilibrium in Game 2-DAL thus dominates not only the alternative diagonally revealing equilibrium but also the equilibria in Games 2 and 2-LAB.

We further discuss the types of out-of-equilibrium messages under different constructions of fully revealing equilibria, which provides some background information for the introduction of additional games in the next subsection. For the equilibria in which one sender reveals only one dimension (or the diagonals), the only out-of-equilibrium messages that may arise are unused messages. Because each sender reveals only a binary characteristic of the state, two messages suffice for each to separate, leaving the other two messages potentially unused. One can, however, have all messages used by prescribing the senders to randomize along the non-equilibrium-relevant dimensions. For the equilibria in which each sender reveals all four states, out-of-equilibrium messages arise as inconsistent message pairs. In such equilibria, the receiver expects to receive messages that indicate the same $(H, V)$. A message pair is therefore out-of-equilibrium when it indicates different entries for $H, V$, or both.

We turn to the single-sender games, in which partitional information is transmitted in equilibrium:

Proposition 2. There exists a partially revealing equilibrium in Game 1, in which the single sender truthfully reveals $(L, U)$ only. The corresponding information partition, $\{\{(L, U)\}$, $\{(R, U),(R, D),(L, D)\}\}$, is the unique informative equilibrium partition. There exists a partially revealing equilibrium in Game 1-DAL, in which the single sender truthfully reveals only along dimension $H$. The corresponding information partition, $\{\{(L, U),(L, D)\},\{(R, U)$, $(R, D)\}\}$, is the unique informative equilibrium partition.

While informative partitions are unique, the two games each have a continuum of equilibrium outcomes, depending on how the receiver randomizes over actions in response to the coarse information. ${ }^{19}$

Note that in our design of Games 1 and 2, removing Sender 2 from Game 2 changes the strategic behavior of Sender 1, while no such change exists between Games 1-DAL and 2-DAL. The importance of the strategic consideration of the other sender's behavior thus varies between the two sets of games.

\footnotetext{
${ }^{19}$ For the equilibrium outcomes in Game 1 , the receiver takes (left, up) in $(L, U)$ and randomizes in the other three states between (right, up) and (right, down) with arbitrary probabilities, leaving out the strictly dominated (left, down). For Game 1-DAL, the receiver randomizes between (left, up) and (left, down) in $(L, U)$ and $(L, D)$, and between (right, up) and (right, down) in $(R, U)$ and $(R, D)$, both with arbitrary probabilities.
} 


\subsection{Three Games with One-Dimensional/Diagonal Messages and Robustness Analysis}

We restrict the message spaces in Games 2 and 2-LAB so that they contain only two messages, creating Games 2-2/M and 2-LAB-2/M. For Game 2-2/M, the message spaces are $M_{1}=\{$ "h"|"left", "right" $\}$ and $M_{2}=\{$ "v"|"up", "down" $\}$. For Game 2-LAB-2/M, Sender 2's message space is the same as that in Game 2-2/M, while Sender 1's becomes $M_{1}=\left\{"(h, v)\right.$ or $\left(h^{\prime}, v^{\prime}\right) " \mid "($ left, up) or (right, down)", "(right, up) or (left, down)" $\}$.

We characterize the equilibria:

Proposition 3. There exists only one class of fully revealing equilibria in Game 2-2/M, in which Sender 1 truthfully reveals along dimension $H$ and Sender 2 along dimension $V$. There exists only one class of fully revealing equilibria in Game 2-LAB-2/M, in which Sender 1 truthfully reveals the diagonals and Sender 2 along dimension V. Any fully revealing equilibrium in the two games is free of out-of-equilibrium beliefs.

Restricting the message spaces serves two experimental purposes. It eliminates the receiver's need to interpret inconsistent messages and thus minimizes strategic uncertainty. It also serves as a step toward controlling the scenarios in which out-of-equilibrium messages arise by first eliminating any out-of-equilibrium messages.

We introduce our last game. Leveraging Ambrus and Takahashi's (2008) insight into the cause of out-of-equilibrium messages under a restricted state space, we eliminate state $(R, D)$ in Game 2-2/M, adjusting the prior so that the remaining three states are equally likely. The result is Game 2-2/M-3/S (Figure 2). For a given fully revealing equilibrium in this game, there is only one profile of out-of-equilibrium messages. A unique scenario is therefore in place in Game 2-2/M-3/S for us to identify observed messages as out-ofequilibrium. Game 2-2/M with no out-of-equilibrium message will then serve as a control for our investigation of the effect of out-of-equilibrium belief specification.

Out-of-equilibrium messages exist in Game 2-2/M-3/S even under the binary message spaces. Consider a deviation by Sender 2 when the state is $(R, U)$. In Game 2-2/M, the receiver, being told by Sender 1, who conforms to the equilibrium, that one component of the state is $R$ and by the deviating Sender 2 that the other component is $D$, cannot detect the deviation. She will take action (right, down) as she will do when $(R, D)$ is truthfully revealed in equilibrium. A deviation does not lead to the receipt of out-of-equilibrium messages because every possible message pair is expected in equilibrium. What deters Sender 2 from deviating is the fact that, in state $(R, U)$, action (right, down) is not as 


\begin{tabular}{|c|ccc|ccc|}
\hline \multicolumn{1}{|c|}{ State: $(L, U)$} \\
\hline Action & \multicolumn{3}{|c|}{ left } & \multicolumn{4}{c|}{ right } \\
\hline up & 20 & 20 & 50 & 0 & 50 & 20 \\
\hline down & 50 & 0 & 10 & 10 & 10 & 0 \\
\hline
\end{tabular}

\begin{tabular}{|c|lcrr|ccc|}
\hline \multicolumn{7}{|c|}{ State: $(R, U)$} \\
\hline Action & \multicolumn{3}{|c|}{ left } & \multicolumn{3}{c|}{ right } \\
\hline up & 0 & 15 & 20 & 20 & 20 & 50 \\
\hline down & 10 & 60 & 0 & 50 & 0 & 10 \\
\hline
\end{tabular}

\begin{tabular}{|c|cccc|ccr|}
\hline \multicolumn{1}{|c|}{ State: $(L, D)$} \\
\hline Action & \multicolumn{3}{|c|}{ left } & \multicolumn{4}{c|}{ right } \\
\hline up & 15 & 0 & 20 & 60 & 10 & 0 \\
\hline down & 20 & 20 & 50 & 0 & 50 & 10 \\
\hline
\end{tabular}

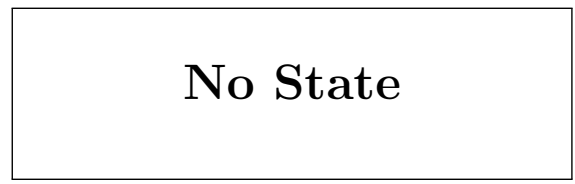

Figure 2: Payoff Profile of Game 2-2/M-3/S

attractive as the equilibrium (right, up).

In Game 2-2/M-3/S, the same deviation creates an entirely different scenario. Given that $(R, D)$ no longer exists, the receiver can detect that there is a deviation because under no circumstance will she receive such a message pair in equilibrium. The deviation does lead to the receipt of out-of-equilibrium messages. To distinguish them from inconsistent message pairs, we refer to the out-of-equilibrium messages that arise due to the restricted state space as irreconcilable message pairs. As mentioned above, for a given fully revealing equilibrium in Game 2-2/M-3/S, there is only one pair of irreconcilable messages. The following proposition states the out-of-equilibrium beliefs required to support the equilibrium:

Proposition 4. There exists only one class of fully revealing equilibria in Game 2-2/M3/S, in which Sender 1 truthfully reveals along dimension $H$ and Sender 2 along dimension $V$. Any fully revealing equilibrium is supported by out-of-equilibrium beliefs that induce the receiver to take action (left, up) with a probability of at least $\frac{4}{5}$ after an irreconcilable message pair is received.

With $(R, D)$ omitted, action (right, down), which can otherwise deter deviations, is strictly dominated for the receiver. Accordingly, (left, up), undominated to the receiver and second least preferred to the senders in $(R, U)$ and $(L, D)$, assumes the task of supporting the equilibrium.

We proceed to our robustness analysis. While our focus is on the games introduced in this subsection, our analysis covers all the two-sender games. We use Battaglini's (2002) robustness criterion and define for each game a corresponding $\varepsilon$-perturbed game: with independent probability $\varepsilon_{i}$ Sender $i$ 's observation of the state is subject to a mistake, in which he observes a random state drawn from a probability distribution, $g_{i}$, that puts positive probabilities on all possible states. The resulting definition of a robust equilibrium is as follows: 
Definition 1 (Battaglini, 2002). An equilibrium is robust if there exists a pair of probability distributions $\left(g_{1}, g_{2}\right)$ and a sequence $\varepsilon^{n}=\left(\varepsilon_{1}^{n}, \varepsilon_{2}^{n}\right)$ converging to zero such that the out-ofequilibrium beliefs of the equilibrium are the limit of the beliefs that the equilibrium strategies would induce in an $\varepsilon$-perturbed game as $\varepsilon^{n} \rightarrow 0$.

We first apply the criterion to Game 2-2/M-3/S:

Corollary 1. None of the fully revealing equilibria in Game 2-2/M-3/S is robust.

Consider an equilibrium in which "left" and "right" are used by Sender 1 to reveal $L$ and $R$ and "up" and "down" are used by Sender 2 to reveal $U$ and $D$. In this equilibrium, ("right", "down") is the irreconcilable, out-of-equilibrium message pair. In an $\varepsilon$-perturbed game, the receiver considers that she has received the message pair after at least one sender's observation of the state was erroneous. When $\varepsilon$ is small, the event that both senders' observations of the state were erroneous is irrelevant; the receiver believes that one of the messages, "right" or "down", conveys information, and in the limit assigns a zero probability to $(L, U)$. In the original, unperturbed Game $2-2 / \mathrm{M}-3 / \mathrm{S}$, the out-ofequilibrium belief required to support the fully revealing equilibrium must, however, put positive probability on $(L, U)$. The consistent belief requirement in Battaglini's (2002) criterion thus rules that the equilibrium is non-robust.

In the games with two-dimensional messages, a fully revealing equilibrium with the senders babbling (for the non-equilibrium-relevant dimensions) by means of randomization is free of out-of-equilibrium beliefs, which makes the equilibrium necessarily robust. However, one can also construct non-robust equilibria, such as those where both senders truthfully reveal all four states. ${ }^{20}$ We thus obtain the following:

Corollary 2. Some, but not all, fully revealing equilibria in Games 2, 2-LAB, and 2-DAL are robust.

In contrast, given that any fully revealing equilibrium in the four-state games with binary messages is free of out-of-equilibrium beliefs, the robustness criterion is trivially satisfied:

\footnotetext{
${ }^{20}$ For an example of non-robust equilibrium, suppose that in equilibrium each sender sends "(left, up)" for state $(L, U)$, "(right, up)" for $(R, U)$, "(left, down)" for $(L, D)$ and "(right, down)" for $(R, D)$. Consider a deviation by Sender 2 in state $(R, D)$ in which he sends "(right, up)". If the receiver responds to the inconsistent message pair, ("(right, down)", "(right, up)"), by taking action (left, up), Sender 2 will be deterred from deviating. However, the fully revealing equilibrium will not be robust: in taking (left, up), the receiver is induced by out-of-equilibrium beliefs that cannot be rationalized as the limit of equilibrium beliefs in a perturbed game.
} 
Corollary 3. All fully revealing equilibria in Games 2-2/M and 2-LAB-2/M are robust.

We conclude our robustness analysis by explaining our choice of criterion. Battaglini's (2002) use of perturbed state observations to impose restrictions on out-of-equilibrium beliefs parallels the consistency requirement of sequential equilibrium, where trembles are introduced at the strategy level. However, the overarching mistake probability for all states renders Battaglini's (2002) criterion stronger than sequential equilibrium, at least for our games. Unless we also require the sequence of completely mixed behavioral strategies to converge to the equilibrium strategies in identical or comparable rates across states, the fully revealing equilibrium in Game 2-2/M-3/S is sequential. Thus, even though our games are finite, using Battaglini's (2002) criterion originally devised for a game with infinite actions allows us to highlight the implausible aspect of the equilibrium in Game 2-2/M-3/S when sequential equilibrium per se has no bite. ${ }^{21}$

\section{Experimental Hypotheses and Procedures}

\subsection{Hypotheses}

The eight games constitute our experimental treatments. We formulate hypotheses about how the treatment variables - the properties of the games - affect information revelation outcomes, i.e., how often receivers identify true states. The hypothesized effects are guided by the equilibrium predictions and other considerations.

Informed by Propositions 1 and 2, we first compare games in which the number of senders is the only treatment variable:

Hypothesis 1. Positive Effect of an Additional Sender: Receivers in Game 2 (2$D A L)$ identify true states more often than do receivers in Game 1 (1-DAL).

\footnotetext{
${ }^{21}$ For sequences of completely mixed strategy profiles in which Sender 1's perturbation probability is orders of magnitude higher in state $(L, U)$ than in $(L, D)$ and Sender 2's in $(L, U)$ than in $(R, U)$, the limit of the receiver's converging beliefs puts probability one on $(L, U)$ after receiving the message pair irreconcilable in equilibrium; the equilibrium is thus sequential. Note, however, that given the receiver's beliefs on and off the equilibrium path, Sender 1, for example, will stand to lose more by trembling in $(L, U)$ (receiving payoff 0$)$ than in $(L, D)$ (receiving payoff 15$)$. It therefore appears to be natural that the perturbation probability should be at least as high in $(L, D)$ as in $(L, U)$. This consideration, which shares the spirit of lower mistake probabilities with costlier mistakes in Myerson's (1978) proper equilibrium, generates a robustness conclusion for Game 2-2/M-3/S that is alternatively reached by Battaglini's (2002) criterion.
} 
Hypothesis 1 is consistent with the key message of Battaglini (2002) that more information can be transmitted with two senders in a multidimensional environment.

Our second hypothesis addresses the treatment effects of dimensional alignments and relabeling. Since the fully revealing equilibrium predicts no outcome difference among Games 2, 2-DAL, and 2-LAB, we do not just consider equilibrium predictions and resort to strategic uncertainty and focality as well. The subject matter of the hypothesis thus departs from that of Battaglini (2002), who considers a pure equilibrium theory.

The comparison between Games 2 and 2-DAL involves two steps. Recall that there are two classes of fully revealing equilibrium in Game 2-DAL: the diagonally revealing and the dimensionally revealing equilibrium. Since the latter dominates the former in terms of less strategic uncertainty, we first select the latter as the one to be compared with the equilibrium in Game 2. Since the dimensionally revealing equilibrium in Game 2-DAL further involves less strategic uncertainty than the fully revealing equilibrium in Game 2, we then hypothesize that the former outperforms the latter as more empirically plausible. We thus obtain the following:

Hypothesis 2a. Positive Effect of Dimensional Alignments: Receivers in Game 2-DAL identify true states more often than do receivers in Game 2.

For Games 2 and 2-LAB, we hypothesize that, despite the comparable degrees of strategic uncertainty from the senders' side, the diagonally revealing equilibrium in Game 2-LAB is less focal than the dimensionally revealing equilibrium in Game 2. We thus have:

Hypothesis 2b. Positive Effect of Focal Revelation Dimensions: Receivers in Game 2 (2-2/M) identify true states more often than do receivers in Game 2-LAB (2$L A B-2 / M)$.

Hypotheses 3 and 4 provide an empirical evaluation of the importance of theoretically robust equilibria that are emphasized in Battaglini (2002) and Ambrus and Takahashi (2008). We first compare games in which the size of message spaces is the only treatment variable. The fully revealing equilibrium again predicts no difference in revelation outcomes between Games 2 (2-LAB) and 2-2/M (2-LAB-2/M). Yet, under the binary message spaces, the equilibria in the latter set of games are free of out-of-equilibrium beliefs and thus robust, while there exist equilibria in the former set that are not. This differentiation informs our next hypothesis:

Hypothesis 3. Positive Effect of Restricting the Message Spaces: Receivers in 
Game 2-2/M (2-LAB-2/M) identify true states more often than do receivers in Game 2 (2-LAB).

Finally, we compare Games 2-2/M and 2-2/M-3/S, in which the number of states is the only treatment variable. Our robustness analysis again informs our hypothesis. The non-robust equilibrium in Game 2-2/M-3/S suggests that the responses of the receiver that invite deviation are more plausible. In translating theoretical plausibility into empirical plausibility, we hypothesize that a plausible response is also a likely response, predicting a lower adherence to fully revealing equilibrium in Game 2-2/M-3/S:

Hypothesis 4. Negative Effect of Restricting the State Space: Receivers in Game 2-2/M identify true state more often than do receivers in Game 2-2/M-3/S.

\subsection{Procedures}

The experiment was conducted in Chinese using z-Tree (Fishchbacher, 2007) at the Taiwan Social Sciences Experimental Laboratory (TASSEL) of the National Taiwan University. Four sessions were conducted for each game using a between-subject design. Each session involved five to seven groups of three (two-sender games) or five to nine groups of two (onesender games), with 492 subjects participating in 32 sessions. Eight sessions were conducted in May 2011 and 24 sessions between June 2012 and January 2013. ${ }^{22}$ Subjects had no prior experience in our experiments and were recruited from the undergraduate/graduate student population of the university.

Upon arrival at the lab, subjects were instructed to sit at separate computer terminals. Each was given a copy of the experimental instructions. Instructions were read aloud and supplemented by slide illustrations. In each session, subjects first participated in three rounds of practice and then 50 official rounds. A random matching protocol with fixed roles was used (repeating partners were allowed).

We illustrate the instructions for two-sender games with two-dimensional messages. Subjects formed groups of three: Member A (Sender 1), Member B (Sender 2), and Member

\footnotetext{
${ }^{22}$ We set a minimum of five groups per session, with the upper bound set by the capacity of TASSEL. Two sessions of Game 1 were conducted in five groups, one in six and one in seven (46 subjects). Two sessions of Game 1-DAL were conducted in seven groups, one in five and one in nine (56 subjects). One session of Game 2 was conducted in seven groups and three in five (66 subjects). For each of Game 2-LAB and Game 2-2/M, one session was conducted in six groups and three in five (63 subjects per each game). All four sessions of Game 2-DAL and Game 2-LAB-2/M were conducted in, respectively, six groups (72 subjects) and five groups (60 subjects). Two sessions of Game 2-2/M-3/S were conducted in six groups and two in five (66 subjects).
} 
$\mathrm{C}$ (receiver). The roles were randomly assigned at the beginning of a session. At the beginning of each round, the computer randomly drew one of $(L, U),(R, U),(L, D)$ or $(R, D)$. The draws were independent across groups and rounds. The drawn outcome would be revealed on the screens of Member A and Member B; they then privately entered their recommendation for Member C. Each sender's recommendation was entered in two steps. Member A entered "left" / "right" first, followed by "up" / "down". The opposite order was used for Member B. After the recommendation, each sender would be asked to make a point prediction about the other's recommendation. The belief elicitation was mildly incentivized with two payoff points for a correct prediction of each dimensional component of the other's recommendation. ${ }^{23}$

The four recommendation inputs, two by each sender, were then revealed to Member $\mathrm{C}$ in one step. Member C's screen would show, for example, that "Member A recommends left; Member A recommends up; Member B recommends right; Member B recommends up." Member C then concluded the round by choosing (left, up), (right, up), (left, down) or (right, down). In every decision step, the corresponding payoff profiles in Figure 1 were shown on each subject's screen. ${ }^{24}$ At the end of each round, subjects were provided with the current round history (the draw, the recommendations of Members A and B, Member C's action, and the subject's own payoff). At the end of the last round, all members were asked to make a point prediction of the state when recommendation "(right, up)" was received from Member A and "(left, down)" from Member B. These pre-specified messages for the prediction were made known to them only at this time. We randomly drew one instance among all groups in the last 30 rounds when these recommendations were observed and rewarded 100 payoff points to the subjects with the correct prediction. ${ }^{25}$

Ten payoff points converted into a real payment of $\mathrm{NT} \$ 5$. A subject was paid his or her sum of rewards from all 50 rounds, including the payoff points from making predictions, plus a NT $\$ 100$ show-up fee. Subjects earned on average NT\$801.78 ( $\approx$ US\$28.06), ranging from $\mathrm{NT} \$ 435$ ( $\approx \mathrm{US} \$ 15.23)$ to $\mathrm{NT} \$ 1,360(\approx \mathrm{US} \$ 47.60)$.

\footnotetext{
${ }^{23}$ This belief elicitation was conducted for all games except Games 1-DAL and 2-DAL. In games with one-dimensional messages, subjects were rewarded with four payoff points for a correct prediction of the other sender's recommendation. Thus, in games with one-dimensional and two-dimensional messages, the maximum payoff points a subject could receive from making a prediction in a round were standardized to four. The simple elicitation with mild incentives was adopted to minimize interference with the major decision tasks.

${ }^{24}$ Refer to online Appendix I (available on the authors' websites) for an English translation (by the authors) of the experimental instructions for Game 2. While the original instructions were in Chinese (online Appendix II), the notation for the state, $(L, U),(R, U),(L, D)$ and $(R, D)$, was used.

${ }^{25}$ This belief elicitation was conducted for all games except Games 1-DAL and 2-DAL. In Games 2-2/M and 2-2/M-3/S, the prediction was for the state when Member A recommended "right" and Member B "down"; in Games 2-LAB-2/M, it was when Member A's recommendation was "(right, up) or (left, down)".
} 


\section{$5 \quad$ Experimental Findings}

The three subsections in this section are structured so that each of them addresses one of our three research questions. Section 5.1 reports findings from the two-dimensional message games, in which we cover the effects of an additional sender, dimensional alignments, and the focality of revelation dimensions. Sections 5.2 and 5.3 report findings from the onedimensional message games; in the former, we examine the effect of restricting the message spaces and, in the latter, we examine the connection between theoretical and empirical implausibility in reference to the specification of out-of-equilibrium beliefs.

In each subsection, we first report one main finding from the quantitative comparisons of revelation outcomes, which helps to evaluate the corresponding hypothesis(es). We then report sub-findings on the key qualitative observations regarding players' strategies, which provide an analysis of what strategies were played that constituted the revelation outcomes.

Table 3 summarizes the main findings.

Table 3: Summary of Main Findings

(Mann-Whitney Tests Comparing Revelation Outcomes)

\begin{tabular}{|c|c|c|c|}
\hline Hypothesis & & Observed \% & $p$-value \\
\hline 1 [Positive Effect of an Additional Sender]: & $F($ Game 2$)>F($ Game 1$)$ & $48 \%$ vs. $39 \%$ & 0.0143 \\
\hline 1 [Positive Effect of an Additional Sender]: & $F($ Game 2-DAL $)>F($ Game 1-DAL $)$ & $73 \%$ vs. $45 \%$ & 0.0143 \\
\hline 2a [Positive Effect of Dimensional Alignments]: & $F($ Game 2 -DAL $)>F($ Game 2$)$ & $73 \%$ vs. $48 \%$ & 0.0143 \\
\hline $2 \mathrm{~b}$ [Positive Effect of Focal Revelation Dimensions]: & $F($ Game 2$)>F($ Game 2-LAB $)$ & $48 \%$ vs. $46 \%$ & $0.4857^{*}$ \\
\hline $2 \mathrm{~b}$ [Positive Effect of Focal Revelation Dimensions]: & $F($ Game $2-2 / \mathrm{M})>F($ Game 2 -LAB-2/M $)$ & $84 \%$ vs. $84 \%$ & $1.0000^{*}$ \\
\hline 3 [Positive Effect of Restricting the Message Spaces]: & $F($ Game $2-2 / \mathrm{M})>F($ Game 2$)$ & $84 \%$ vs. $48 \%$ & 0.0143 \\
\hline 3 [Positive Effect of Restricting the Message Spaces]: & $F($ Game 2 -LAB-2/M $)>F($ Game 2 -LAB $)$ & $84 \%$ vs. $46 \%$ & 0.0143 \\
\hline 4 [Negative Effect of Restricting the State Space]: & $F^{\prime}($ Game $2-2 / \mathrm{M})>F^{\prime}($ Game $2-2 / \mathrm{M}-3 / \mathrm{S})$ & $84 \%$ vs. $50 \%$ & 0.0143 \\
\hline
\end{tabular}

Note: $F($ Game $\mathrm{X})$ refers to the last-30-round aggregate frequency of state-action agreements in Game X, with which we measure how often receivers identified true states by recording the instances in which their ideal actions were taken. $F^{\prime}($ Game $\mathrm{X}$ ) refers to the last-30-round aggregate frequency of state-message-action agreements in Game X. Such a measure is used for the comparison between Games 2-2/M and 2-2/M-3/S, because state-action agreements do not provide a common ground for comparing a three-state game with a four-state game. Refer to Table 7 in Appendix $\mathrm{C}$ for a more comprehensive summary statistics. * indicates that the $p$-value is two-sided. For the Mann-Whitney tests, we consider a comparison result as being statistically significant if and only if the one-sided $p \leqslant 0.0571$.

\subsection{Additional Sender, Dimensional Alignments, and Focality}

\section{Finding 1 (Outcomes).}

- Positive Effect of an Additional Sender: Receivers in Game 2 (2-DAL) identified true states significantly more often than did receivers in Game 1 (1-DAL).

- Positive Effect of Dimensional Alignments: Receivers in Game 2-DAL identified true states significantly more often than did receivers in Game 2. 
- No Effect of Focal Revelation Dimensions: Receivers in Game 2-LAB identified true states as often as did receivers in Game 2.

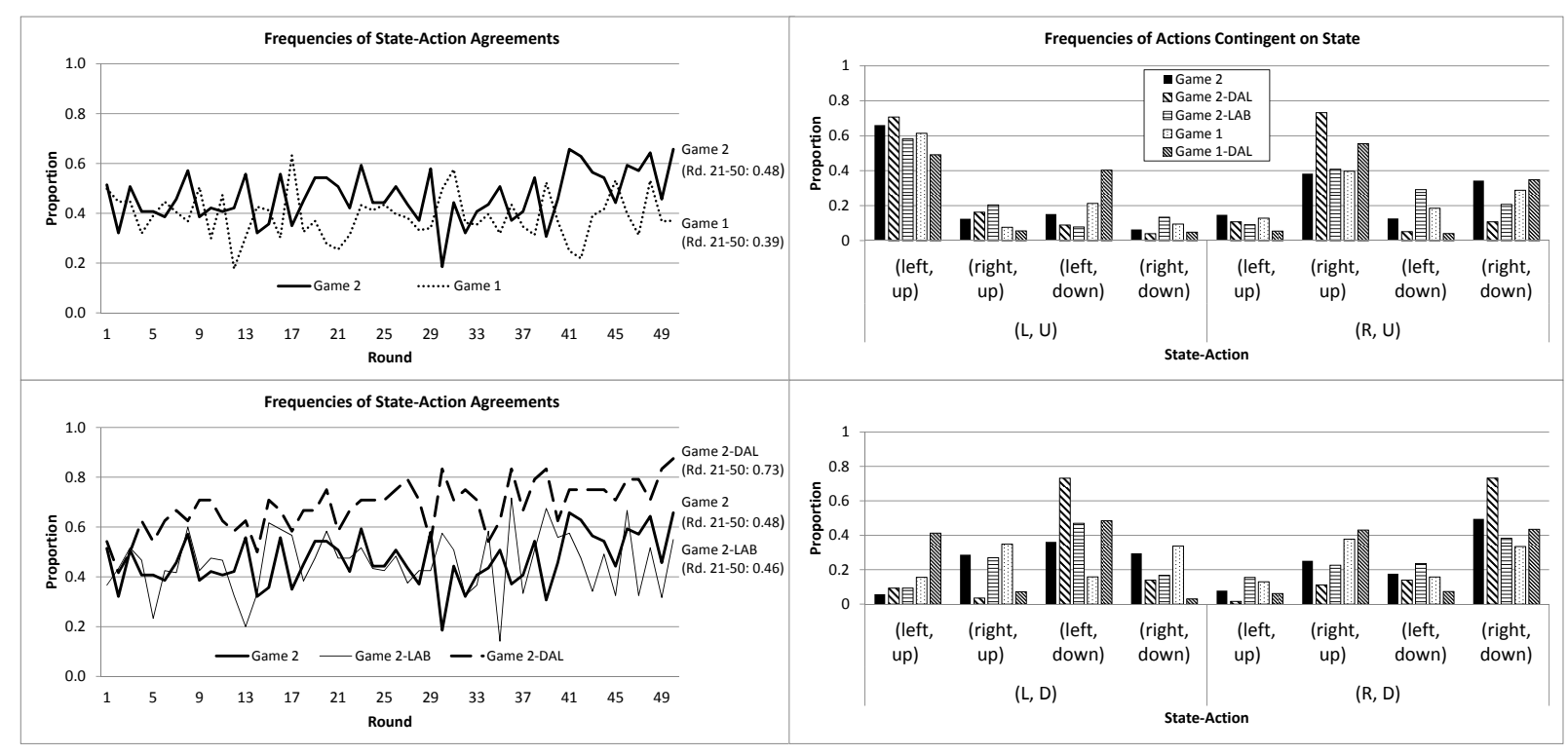

(a) Frequencies of State-Action Agreements

(b) Frequencies of Actions Contingent on State

Figure 3: Information Revelation Outcomes in Games 2, 2-DAL, 2-LAB, 1, and 1-DAL

Our findings indicate that information transmission facilitated receivers' identifications of true states and, to varying degrees depending on whether all-state dimensional alignments were present, two senders served better than one. Figure 3(a) presents the frequencies of state-action agreements, with which we measure how often receivers identified true states by recording the instances in which their ideal actions were taken. The frequency aggregated across the last 30 rounds of all sessions was $48 \%$ (73\%) in Game 2 (2-DAL), which is significantly higher than the 39\% (45\%) in Game 1 (1-DAL). This comparison result confirms Hypothesis 1 ( $p=0.0143$, Mann-Whitney tests $){ }^{26}$ All frequencies were significantly higher than $25 \%$ ( $p=0.0625$, Wilcoxon signed-rank tests), which is the benchmark for no information transmission with ideal actions taken as a result of random guesses. ${ }^{27}$ (For the Mann-Whitney tests, we consider a comparison result as being statistically significant if

${ }^{26}$ Refer to Figure 12 in Appendix C for the frequency comparison between Games 2-DAL and 1-DAL.

${ }^{27}$ All of our statistical tests use aggregate data from the last 30 rounds of each session as an independent observation. Further convergence in varying degrees across games, which deepens the comparisons in favor of our hypotheses, was typically observed after the 30th round. (In Game 2, for example, the agreement frequency in the last 10 rounds was $10 \%$ higher at 58\%.) The 30-round cutoff, though rather arbitrary, is adopted with a view to balancing conservativeness with convergence. Table 7 in Appendix $\mathrm{C}$ contains statistics under three different aggregations (the first 20, the last 30, and the last 10 rounds). From now on, all frequencies reported and referred to are from the last 30 rounds. 
and only if the one-sided $p \leqslant 0.0571$. For the Wilcoxon signed-rank tests, the one-sided $p=0.0625$, which is the lowest possible for four observations, is adopted as the threshold.)

The frequency in Game 2-DAL was in turn significantly higher than that in Game 2, which confirms Hypothesis 2a ( $p=0.0143$, Mann-Whitney test). Figure 3(b) breaks down the frequencies for each state. Naturally, the dimensional alignments in Game 2-DAL improved revelation outcomes primarily through $(L, D)$ and $(R, U)$, the states in which no alignment is in place for, respectively, Sender 1 and Sender 2 in Game 2.

There was, however, no significant difference between Games 2 and 2-LAB, which rejects Hypothesis $2 \mathrm{~b}$ (two-sided $p=0.4857$, Mann-Whitney test). The less focal revelation dimensions in Game 2-LAB did not adversely affect revelation outcomes, although compared to Game 2 a lower degree of convergence was observed. ${ }^{28}$

Figure 3(b) also shows that, for Games 1 and 2, the more frequent state-action agreements in the latter originated from $(L, D)$ and $(R, D)$. On the other hand, for Games 1-DAL and 2-DAL, the more frequent agreements in the latter were observed throughout all states. The qualitative nature of this "difference-in-difference" suggests that the addition of Sender 2 influenced behavior differently with or without all-state dimensional alignments. This is consistent with the fact that the equilibrium strategy of the singlesender is different between Games 1 and 1-DAL.

We next explore the strategies played in the two-sender games:

\section{Finding 1a (Strategies in Two-Sender Games).}

- Senders in the two-sender games with two-dimensional messages, Games 2, 2-DAL, and 2-LAB, revealed along their equilibrium-relevant dimensions and randomized along the other dimensions, except for the states without dimensional alignment in Games 2 and 2-LAB.

- Receivers in Game 2-DAL followed recommendations according to the equilibriumrelevant dimensions. Receivers in Games 2 and 2-LAB followed recommendations less often for those messages that could have come from states without dimensional alignment, unless the two senders made the same recommendation.

Our findings indicate that the senders' behavior in states with dimensional alignment was consistent with the prescriptions of fully revealing equilibrium. Using the literal rec-

\footnotetext{
${ }^{28}$ The state-action agreement frequencies in the last 10 rounds indicate further convergence in Game 2 $(58 \%)$ but not in Game 2-LAB (46\%), with the former significantly higher ( $p=0.0143$, Mann-Whitney test). Our no-effect conclusion rejecting Hypothesis $2 \mathrm{~b}$ is drawn in adherence to the criterion of the last 30 round data commonly applied to all other comparisons.
} 


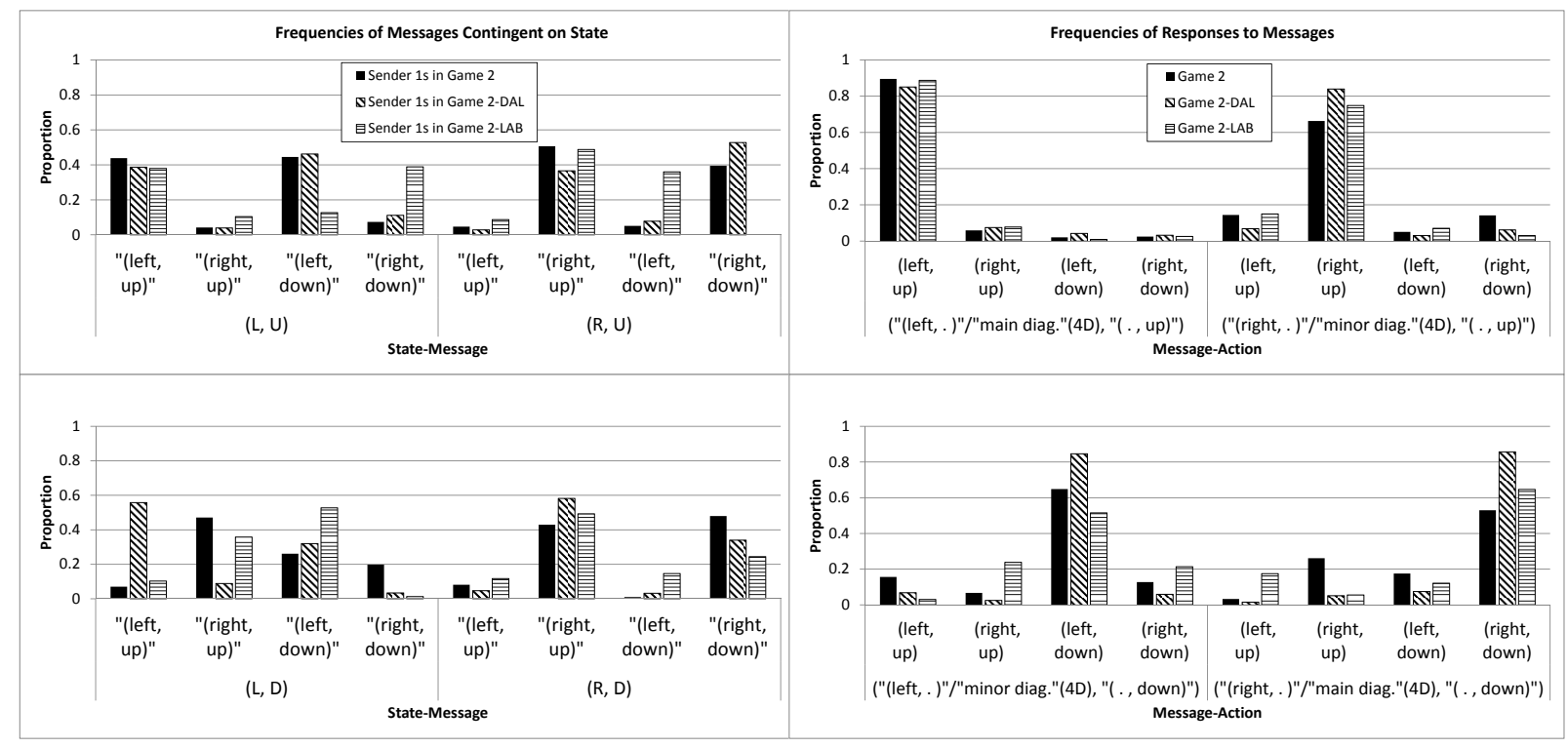

(a) Frequencies of Messages Contingent on State (Sender 1s)

(b) Frequencies of Responses to Message Pairs

Figure 4: Strategies in Games 2, 2-DAL, and 2-LAB

ommendations, senders revealed only along their equilibrium-relevant dimensions, including the diagonals in Game 2-LAB ${ }^{29}$ Figure 4(a) presents the message uses in each state, where for clarity we include Sender 1s' messages only. ${ }^{30}$ To see the consistency with the equilibrium prescriptions, consider the message uses in $(L, U)$, a representative state with dimensional alignment. Messages "(left, up)" and "(left, down)" constituted 85\% or more of Sender 1s' messages in Games 2 (44\% and 45\%) and 2-DAL (39\% and 46\%); "(left, up)" and "(right, down)" constituted $77 \%$ of Sender 1s' messages in Game 2-LAB (38\% and $39 \%$ ). For Sender 2s' messages, "(left, up)" and "(right, up)" were used at least $78 \%$ of the time in Games 2 (35\% and 45\%), 2-DAL (45\% and 47\%), and 2-LAB (37\% and 41\%).

Different behavioral patterns were, however, observed in states without dimensional alignment. The three most frequently used messages were the truthful recommendation and two messages that are inconsistent with the equilibrium-relevant dimensions (in reference to the literal meanings and the message uses in states with alignment). Among them, the most frequent was the recommendation for the senders' own ideal actions. For instance, in $(L, D)$

\footnotetext{
${ }^{29}$ Corresponding to the fact that meanings in cheap-talk games are determined in equilibrium, it is the observed uses of messages that determine how meanings should be assigned in our findings. As an anchoring point for interpreting observed behavior, we nevertheless presume that subjects transmit information using the literal meanings of recommendations, in which "recommend $(h, v)$ " is considered to mean "it is in your best interest to take $(h, v)$." Deviations are interpreted accordingly.

${ }^{30}$ Figure 4(b) presents the receivers' responses to messages; for Game 2-LAB, "main diagonal" refers to either "(left, up)" or "(right, down)" and "minor diagonal" to "(right, up)" or "(left, down)". Figure 13(a) in Appendix C presents Sender 2s' message uses.
} 
in Game 2, in which Sender 1's ideal action is (right, up), Sender 1s' messages concentrate on "(left, down)", "(right, up)", and "(right, down)", with respective frequencies 26\%, 47\%, and 20\%. ${ }^{31}$ With Sender 2s' messages concentrated on "(left, down)" and "(right, down)" (43\% and 45\%) in $(L, D)$, which was accurately anticipated by Sender 1s, Sender 1s' self-serving recommendation of "(right, up)" resulted in message pair ("(right, up)", "(left, down)") or ("(right, up)", "(right, down)"). ${ }^{32}$ Whether Sender 1s' deviation intended to induce their own ideal actions would be rewarded or punished depended on how receivers would interpret these inconsistent messages.

Table 4: Receivers' Responses to All Message Pairs

\begin{tabular}{lcccc}
\hline$m_{1} \backslash m_{2}$ & "(left, up)" & "(right, up)" & "(left, down)" & "(right, down)" \\
\hline \multicolumn{5}{c}{ A. Game 2 } \\
\hline "(left, up)" & $(\mathbf{0 . 8 8}, 0.12,0.00,0.00)$ & $(\mathbf{0 . 8 9}, 0.03,0.03,0.05)$ & $(0.20,0.10, \mathbf{0 . 4 5}, 0.25)$ & $(0.39,0.00, \mathbf{0 . 4 3}, 0.17)$ \\
"(right, up)" & $(0.29, \mathbf{0 . 2 9}, 0.04,0.38)$ & $(0.08, \mathbf{0 . 9 2}, 0.00,0.00)$ & $(0.07,0.33,0.08, \mathbf{0 . 5 2})$ & $(0.02,0.08,0.47, \mathbf{0 . 4 3})$ \\
"(left, down)" & $(\mathbf{1 . 0 0}, 0.00,0.00,0.00)$ & $(\mathbf{0 . 8 3}, 0.10,0.03,0.03)$ & $(0.00,0.03, \mathbf{0 . 9 7}, 0.00)$ & $(0.07,0.14, \mathbf{0 . 6 4}, 0.14)$ \\
"(right, down)" & $(0.19, \mathbf{0 . 5 0}, 0.06,0.25)$ & $(0.09, \mathbf{0 . 7 3}, 0.09,0.09)$ & $(0.01,0.62,0.04, \mathbf{0 . 3 3})$ & $(0.01,0.03,0.06, \mathbf{0 . 9 0})$ \\
\hline \multicolumn{5}{c}{ B. Game 2-DAL } \\
\hline "(left, up)" & $(\mathbf{0 . 9 7}, 0.00,0.03,0.00)$ & $(\mathbf{0 . 9 2}, 0.00,0.05,0.03)$ & $(0.08,0.00, \mathbf{0 . 8 3}, 0.10)$ & $(0.06,0.03, \mathbf{0 . 8 7}, 0.04)$ \\
"(right, up)" & $(0.02, \mathbf{0 . 8 9}, 0.02,0.07)$ & $(0.03, \mathbf{0 . 9 0}, 0.03,0.03)$ & $(0.00,0.08,0.12, \mathbf{0 . 8 0})$ & $(0.04,0.11,0.04, \mathbf{0 . 8 0})$ \\
"(left, down)" & $(\mathbf{0 . 7 6}, 0.13,0.04,0.07)$ & $(\mathbf{0 . 8 3}, 0.12,0.04,0.02)$ & $(0.03,0.03, \mathbf{0 . 9 5}, 0.00)$ & $(0.06,0.03, \mathbf{0 . 8 5}, 0.06)$ \\
"(right, down)" & $(0.11, \mathbf{0 . 7 5}, 0.05,0.09)$ & $(0.10, \mathbf{0 . 8 2}, 0.02,0.06)$ & $(0.00,0.03,0.15, \mathbf{0 . 8 2})$ & $(0.03,0.00,0.00, \mathbf{0 . 9 7})$ \\
\hline & \multicolumn{5}{c}{$\mathbf{C . \mathbf { G a m e ~ 2 - L A B }}$} \\
\hline "(left, up)" & $(\mathbf{0 . 9 7}, 0.03,0.00,0.00)$ & $(\mathbf{0 . 8 9}, 0.11,0.00,0.00)$ & $(0.32,0.08,0.20, \mathbf{0 . 4 0})$ & $(0.27,0.12,0.19, \mathbf{0 . 4 2})$ \\
"(right, up)" & $(0.32, \mathbf{0 . 4 0}, 0.20,0.08)$ & $(0.13, \mathbf{0 . 8 5}, 0.00,0.03)$ & $(0.01,0.09, \mathbf{0 . 4 3}, 0.47)$ & $(0.07,0.29, \mathbf{0 . 4 6}, 0.18)$ \\
"(left, down)" & $(0.22, \mathbf{0 . 5 7}, 0.17,0.04)$ & $(0.03, \mathbf{0 . 9 4}, 0.03,0.00)$ & $(0.00,0.02, \mathbf{0 . 9 1}, 0.07)$ & $(0.02,0.55, \mathbf{0 . 3 0}, 0.14)$ \\
"(right, down)" & $(\mathbf{0 . 9 6}, 0.04,0.00,0.00)$ & $(\mathbf{0 . 7 7}, 0.10,0.03,0.10)$ & $(0.14,0.03,0.14, \mathbf{0 . 6 9})$ & $(0.00,0.00,0.00, \mathbf{1 . 0 0})$ \\
\hline
\end{tabular}

Note: Each array of numbers represents the frequencies of (left, up), (right, up), (left, down), (right, down). The numbers in bold refer to the cases when receivers follow Sender 1s' recommendation $\left(m_{1}\right)$ on dimension $H$ (diagonals) and Sender 2s' recommendation $\left(m_{2}\right)$ on dimension $V$.

Table 4 presents the receivers' responses to all message pairs. Consider as a contrasting case the responses in Game 2-DAL after messages ("(right, up)", "(left, down)") or ("(right, up)", "(right, down)"). In each case, action (right, down) was taken $80 \%$ of the time. If receivers in Game 2 had filtered the message pairs in the same manner by following "right" from Sender 1's recommendation and "down" from Sender 2's recommendation, Sender 1s' deviation would have been met with a severe punishment of zero payoff. What was observed, however, was that the receivers' responses in Game 2 put substantial frequencies either on Sender 1's ideal (right, up) [33\%, after ("(right, up)", "(left, down)")] or on the harmless

\footnotetext{
${ }^{31}$ Similar patterns were observed in other states without alignment. Messages "(right, down)", "(right, up)", and "(left, down)" constituted $88 \%$ of Sender 1s' messages in $(R, D)$ in Game 2-LAB. For Sender $2 \mathrm{~s}$ in $(R, U)$, "(right, up)", "(left, down)", and "(right, down)" constituted $88 \%$ of messages in Game 2 and $89 \%$ in Game 2-LAB. In each case, the most frequent messages were the recommendations for the senders' own ideal actions.

${ }^{32}$ Sender 1s' predicted frequencies for Sender $2 \mathrm{~s}$ ' messages in $(L, D)$ were $55 \%$ for "(left, down)" and $41 \%$ for "(right, down)". Overall, senders' prediction of the other senders' messages was consistent with the actual message uses. Figure 14(a) in Appendix C presents the predictions in Games 2 and 2-LAB.
} 
(left, down) [(47\%, after ("(right, up)", "(right, down)")]. Thus, despite incongruence with the prescriptions of fully revealing equilibrium, the senders' behavior in states without dimensional alignment reflected the receivers' less severely punishing and at times rewarding responses to inconsistent messages. ${ }^{33}$

Turning to the overall behavior of receivers, we note that, consistent with the prescriptions of fully revealing equilibrium given the actual message uses, receivers in Game 2-DAL took $(h, v)$ with frequencies at least $75 \%$ when (" $(h,$.$) ", "(. , v)") were received. This result$ was observed even when the two messages were totally inconsistent, which suggests that subjects had the sophistication to filter message pairs according to the equilibrium-relevant dimensions.

With the senders' deviations in states without dimensional alignment, receivers in Games 2 and 2-LAB behaved differently from those in Game 2-DAL. The observed behavior can be organized by a response rule in which messages are filtered in two different ways: receivers followed senders according to the equilibrium-relevant dimensions when no message that could have come from states without alignment was received; when one was received, receivers followed the relevant dimensions less often unless the message was endorsed by an identical message from the other sender. The receivers' responses in Game 2 when (right, up) was received from Sender 1s provide a representative example. Receivers took (right, up) with $29 \%$ frequency if "(left, up)" was received from Sender 2s; (right, down) with $52 \%$ frequency if "(left, down)" was received from Sender 2s; (right, down) with $43 \%$ frequency if "(right, down)" was received from Sender 2s; and "(right, up)" with frequency $92 \%$ if "(right, up)" was also received from Sender 2s. By contrast, when any message pair that forms ("(left, . )", “( . , up)") was received, (left, up) was taken with $83 \%-100 \%$ frequency.

The findings above address our first research question. In the two-dimensional message environment, how receivers responded to inconsistent messages was crucial to the information transmission outcome. Furthermore, the presence or absence of all-statement dimensional alignments played an important role. In Game 2-DAL, the all-state dimensional alignments presented receivers with minimal strategic uncertainty regarding how to

\footnotetext{
${ }^{33}$ In Game 2, the other less frequent deviating message, "(right, down)", induced a mixture of higher rewards and more severe punishments. Overall in games without all-statement dimensional alignments, the three frequently sent messages in a given state without dimensional alignment gave senders comparable expected payoffs (calculated based on the observed strategies of other senders and receivers), with sometimes even higher payoffs for the deviating messages. For Sender 1s in $(L, D)$, expected payoffs from "(left, down)", "(right, up)", and "(right, down)" were 21.33, 21.22, and 22.95 in Game 2; for Sender 1s in $(R, D)$, payoffs from "(right, down)", "(right, up)", and "(left, down)" were 19.06, 22.33, and 26.08 in Game 2-LAB; for Sender 2s in $(R, U)$, payoffs from "(right, up)", "(left, down)", and "(right, down)" were 19.97, 16.6, and 20.66 in Game 2 and 18.19, 31.52 and 33.45 in Game 2-LAB.
} 
interpret inconsistent messages, which in turn fostered senders' adherence to reveal according to their equilibrium-relevant dimensions. In Games 2 and 2-LAB, where senders' ideal actions lie across the equilibrium-relevant dimensions in states without alignment, receivers followed the recommendations less often and were not as predictable with inconsistent messages. This behavior in turn made senders' deviations more justifiable. While subjects' strategies followed the logic of Battaglini's (2002) equilibrium, the extent of the adherence depended on the nature of dimensional interest alignments embodied in the structure of the game. This result represents a difference between our findings and the theory of Battaglini (2002) in which a dimension of common interest is endogenous.

We conclude this subsection by analyzing the strategies in the two one-sender games:

\section{Finding 1b (Strategies in One-Sender Games).}

- Senders in Game 1-DAL revealed on dimension H as did Sender 1s in Game 2-DAL; Senders in Game 1 behaved differently from Sender 1s in Game 2, consistent with the partially revealing equilibrium in which only $(L, U)$ is revealed.

- Receivers in Game 1-DAL followed the senders' recommendations only for dimension $H$; the receivers' responses in Game 1 reflected the senders' revelations of $(L, U)$.

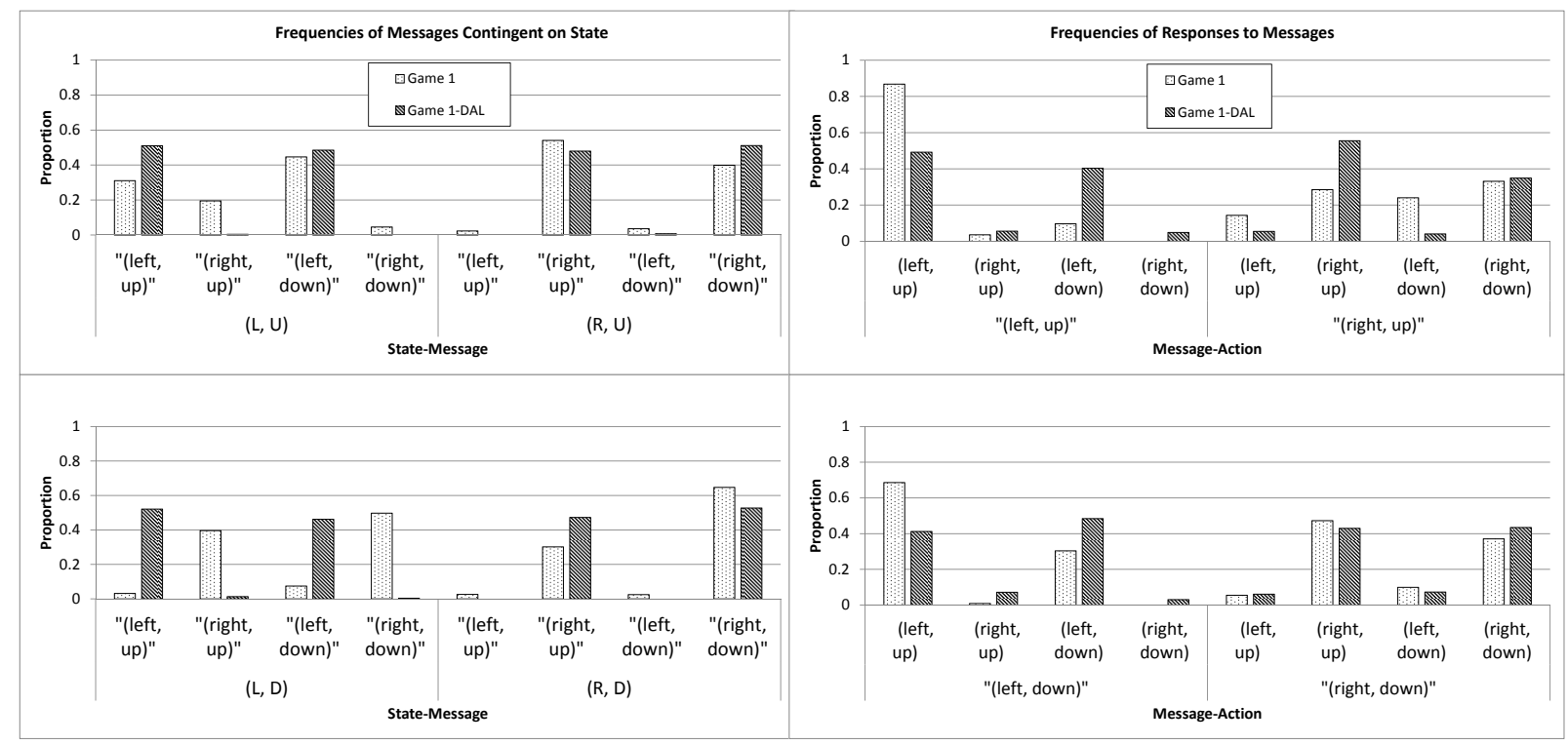

(a) Frequencies of Messages Contingent on State

(b) Frequencies of Responses to Messages

Figure 5: Strategies in Games 1 and 1-DAL

Figure 5 presents the message uses and responses in the two one-sender games. Similar to Sender 1s in Game 2-DAL, senders in Game 1-DAL revealed on dimension H. In Game 
1, the combined frequency of "(left, up)" and "(left, down)" was $76 \%$ in $(L, U)$ and never exceeded $10 \%$ in the other three states, across which the uses of "(right, up)" and "(right, down)" were fairly uniform. These message uses resulted in the revelation of $(L, U)$, with slight or no information provided for the other three states, consistent with the partially revealing equilibrium. ${ }^{34}$

In Game 1-DAL, receivers listened to senders on dimension $H$, mostly ignoring the part of messages for dimension $V$. In Game 1, the receivers' responses to "(left, up)" and "(left, down)" were most often (left, up), largely consistent with the finding that the two messages were used to reveal $(L, U)$, a pattern not observed in Game 1-DAL. ${ }^{35}$

\subsection{Restricted Message Spaces}

Finding 2 (Outcomes). Positive Effect of Restricting the Message Spaces: Receivers in Game 2-2/M (2-LAB-2/M) identified true states significantly more often than did receivers in Game 2 (Game 2- $L A B$ ).

Restricting the message spaces increased overall state-action agreement frequencies by as much as $80 \%$. Figure 6 presents the information revelation outcomes in the two onedimensional message games, along with their four-message counterparts for comparisons. The frequency of state-action agreements in Game 2-2/M (Game 2-LAB-2/M) was 84\% (84\%), which is significantly higher than the $48 \%$ (46\%) in Game 2 (Game 2-LAB). This comparison result confirms Hypothesis 3 ( $p=0.0143$, Mann-Whitney tests). ${ }^{36}$ Under the binary message spaces, high frequencies of state identifications $(76 \%-95 \%)$ were observed for all states. The states without dimensional/diagonal alignment were still discernable; as in Games 2 and 2-LAB, state identifications in Games 2-2/M and 2-LAB-2/M were more frequent in states with alignment than in those without. However, it was in the states with

\footnotetext{
${ }^{34}$ The frequencies of $(L, U)$ contingent on "(left, up)" and "(left, down)" were, respectively, $81 \%$ and $78 \%$. Contingent on "(right, up)", the frequency of $(R, U)$ was higher than that of $(R, D)(38 \%$ vs. $19 \%)$, and vice versa for "(right, down)" (27\% vs. $38 \%)$, while the two frequencies of $(L, D)$ were very close $(28 \%$ and 31\%). Refer to Figure 13(b) in Appendix C for the conditional distributions of states implied by the message uses.

${ }^{35}$ Note that our findings from the single-sender games contrast with those from other single-sender communication game experiments, in which lying aversion (the over-transmission of information in reference to equilibrium predictions) is frequently documented (e.g., Dickhaut et al., 1995; Blume, et al., 1998, 2001; Gneezy, 2005; Cai and Wang, 2006; Kawagoe and Takizawa, 2009). We speculate that the opportunity to provide truthful information in equilibrium for one dimension or for one state allowed subjects to avoid outright lying while still behaving as predicted.

${ }^{36}$ The less focal revelation dimensions had virtually zero effect when the message spaces were restricted. The part of Hypothesis 2b comparing Games 2-2/M and Game 2-LAB-2/M is rejected with an extreme two-sided $p=1$ from the Mann-Whitney test.
} 


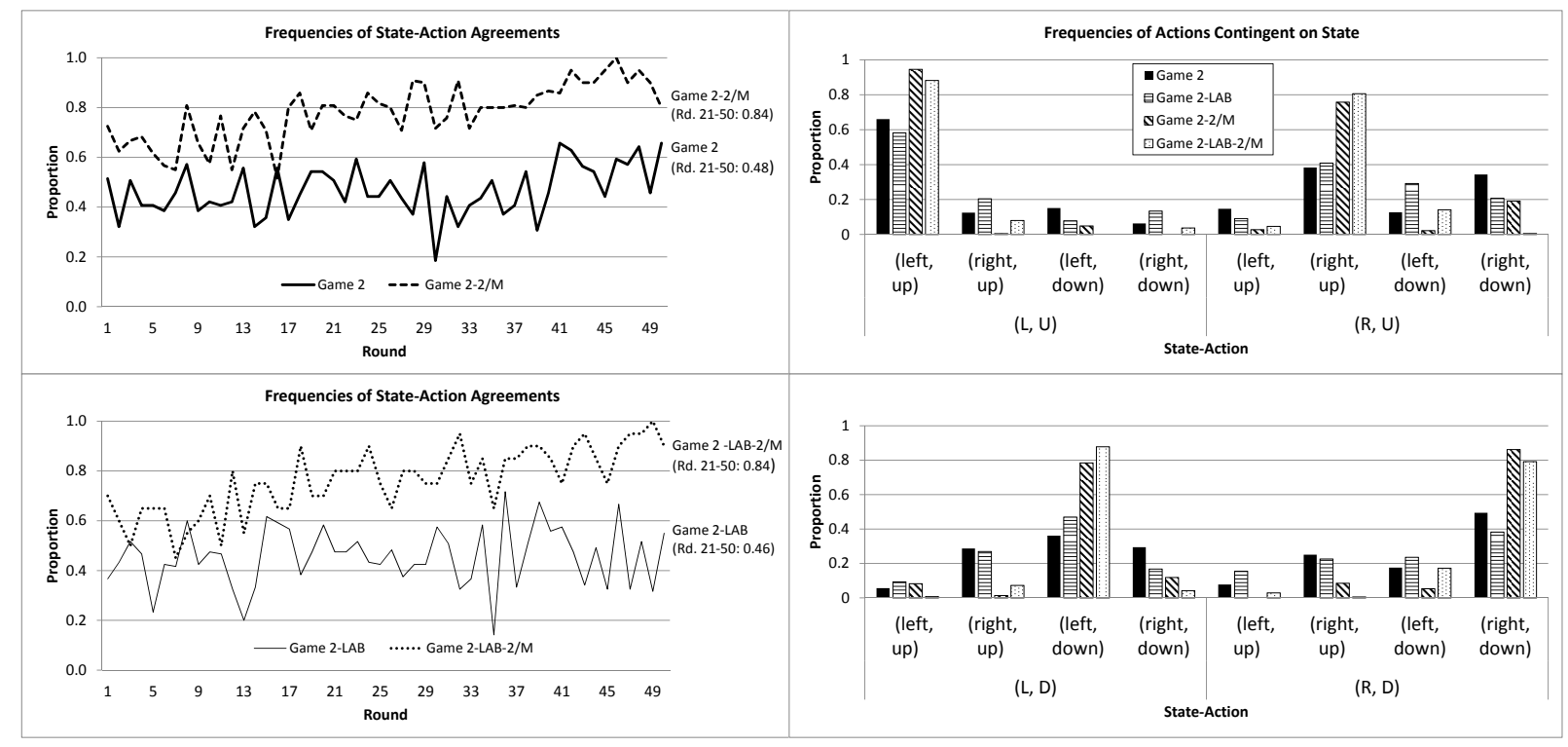

(a) Frequencies of State-Action Agreements

(b) Frequencies of Actions Contingent on State

Figure 6: Information Revelation Outcomes in Games 2, 2-LAB, 2-2/M, and 2-LAB-2/M

no alignment that restricting the message spaces showed a slightly stronger effect. ${ }^{37}$

Addressing our second research question, the findings above suggest that restricting the message spaces substantially increased the frequencies of full revelation outcomes, and the effect was exerted especially on states without dimensional alignment.

\section{Finding 2a (Strategies).}

- Senders in the two-sender games with one-dimensional messages, Games 2-2/M and 2-LAB-2/M, behaved in high accord with the prescriptions of fully revealing equilibrium, even for states without dimensional/diagonal alignment.

- Receivers in the two games virtually always followed the senders' recommendations.

Figure 7 presents the message uses in Games 2-2/M and 2-2/M-LAB. ${ }^{38}$ Message uses consistent with the prescriptions of fully revealing equilibrium were observed with $92 \%-$ $99 \%$ frequency in states with alignment. In states without alignment, adherence was observed with $79 \%-89 \%$ frequency, compared to $33 \%-43 \%$ when four messages were

${ }^{37}$ The positive differences of Game 2-2/M over Game 2 in state identifications were $42 \%$ and $38 \%$ for $(L, D)$ and $(R, U)$ and $37 \%$ and $29 \%$ for $(R, D)$ and $(L, U)$. For Games 2-LAB-2/M and 2-LAB, the differences were $41 \%$ and $40 \%$ for $(R, D)$ and $(R, U)$ and $41 \%$ and $30 \%$ for $(L, D)$ and $(L, U)$.

${ }^{38}$ Given that the messages are binary, for each state we present only the frequencies of one message (truthful dimensional recommendation). For the two-dimensional message games included for comparison, we condense the different message pairs accordingly. For Game 2-LAB-2/M, "main diagonal" refers to message "(left, up) or (right, down)" and "minor diagonal" to "(right, up) or (left, down)". 


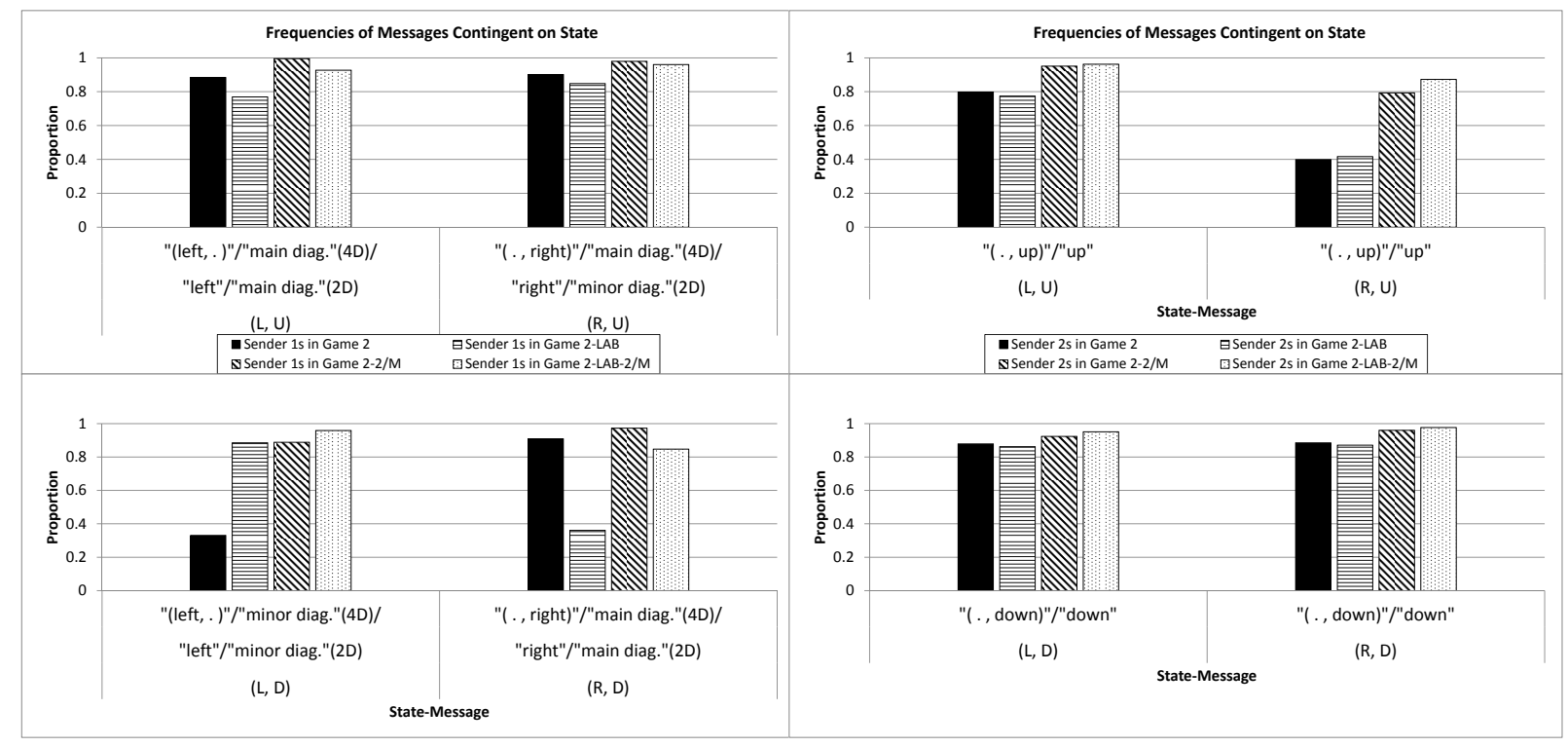

(a) Frequencies of Messages Contingent on State: Sender 1s

(b) Frequencies of Messages Contingent on State: Sender $2 \mathrm{~s}$

Figure 7: Senders' Strategies in Games 2, 2-LAB, 2-2/M, and 2-LAB-2/M

available. Under the binary message environment, the senders' deviating recommendations intended to induce their own ideal actions could only be made for one dimension, and they were very likely to be severely punished given that the other sender recommended truthfully on the other dimension.

Figure 8 confirms that the receivers' responses were highly predictable under the binary message environment. The frequencies for following recommendations were at least $91 \%$ and as high as $100 \%$. The ways in which message pairs are combined are different for Games 2-2/M and 2-LAB-2/M. In Game 2-LAB-2/M, guided by Sender 2s' dimensional messages, receivers eliminate an irrelevant component in Sender 1s' diagonal messages. In Game 2-2/M, with dimensional messages from both senders, only a simple combination of messages is required. We illustrate with our last set of findings below that even such an apparently simple task of combining messages was backed by the receivers' considerations of the senders' incentives.

\subsection{Restricted State Space: Theoretical and Empirical Implau- sibility}

Finding 3 (Outcomes). Negative Effect of Restricting the State Space: Receivers in Game 2-2/M-3/S identified true states significantly less often than did receivers in Game 


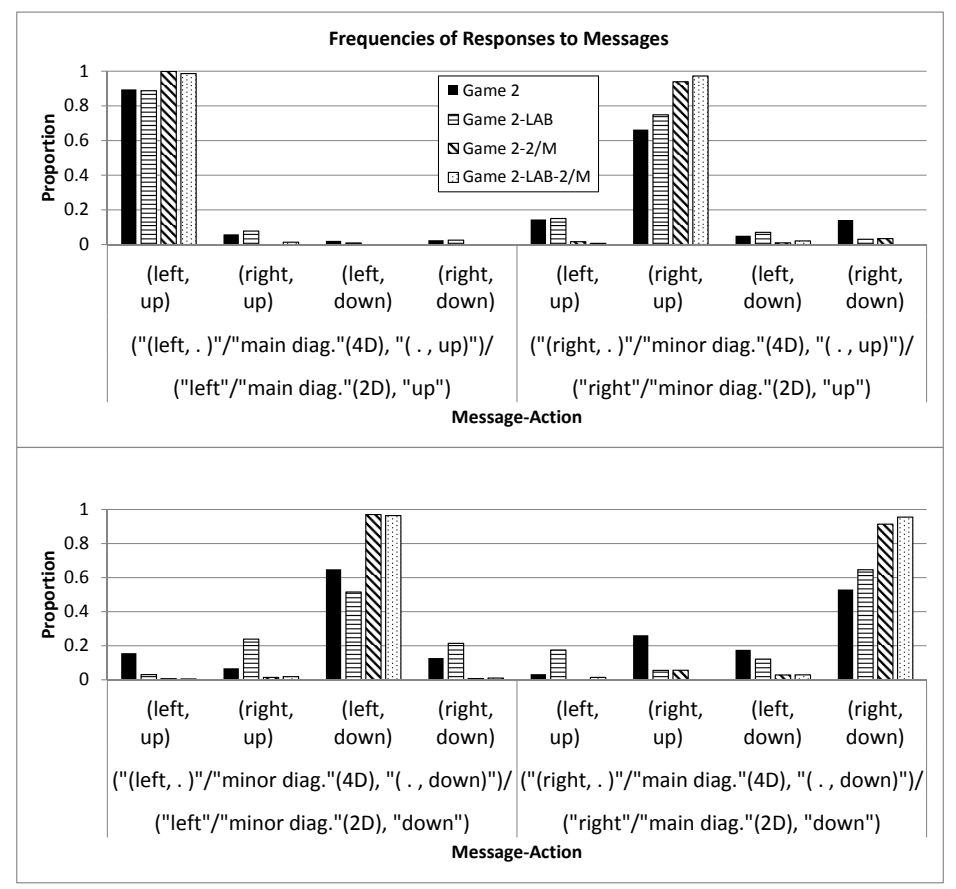

Figure 8: Receivers' Responses in Games 2, 2-LAB, 2-2/M, and 2-LAB-2/M

2-2/M.

The omission of a state, with its robustness implication for the fully revealing equilibrium in Game 2-2/M-3/S, significantly reduced the instances in which receivers identified true states. Figure 9(a), upper panel, presents the frequencies of state-message-action agreements, an alternative measure that we use to compare the revelation outcomes in Game 2-2/M-3/S with those in Game 2-2/M. ${ }^{39}$ The frequency in Game 2-2/M-3/S was $50 \%$, which is significantly lower than the $84 \%$ in Game 2-2/M. This comparison result confirms Hypothesis $4(p=0.0143$, Mann-Whitney test $){ }^{40}$ Figure $9(\mathrm{~b})$ shows that less frequent state identifications were observed in all three states.

\section{Finding 3a (Strategies).}

- Senders in Game 2-2/M-3/S deviated from the message uses observed in Game 2-2/M in states without dimensional alignment.

\footnotetext{
${ }^{39}$ The measure using state-action agreements, which is presented in the lower panel, does not provide a common ground for comparing a three-state game with a four-state game, because the probability of receivers taking ideal actions out of random guesses is higher in Game 2-2/M-3/S. A condition for the validity of the new measure is that the literal meanings of the recommendations are used, which was observed in Game 2-2/M.

${ }^{40}$ The same qualitative difference with statistical significance was also observed using state-action agreements, even though the measure favors Game 2-2/M-3/S. The frequency is $84 \%$ in Game $2-2 / \mathrm{M}$, which is significantly higher than the $67 \%$ in Game 2-2/M-3/S ( $p=0.0571$, Mann-Whitney test).
} 


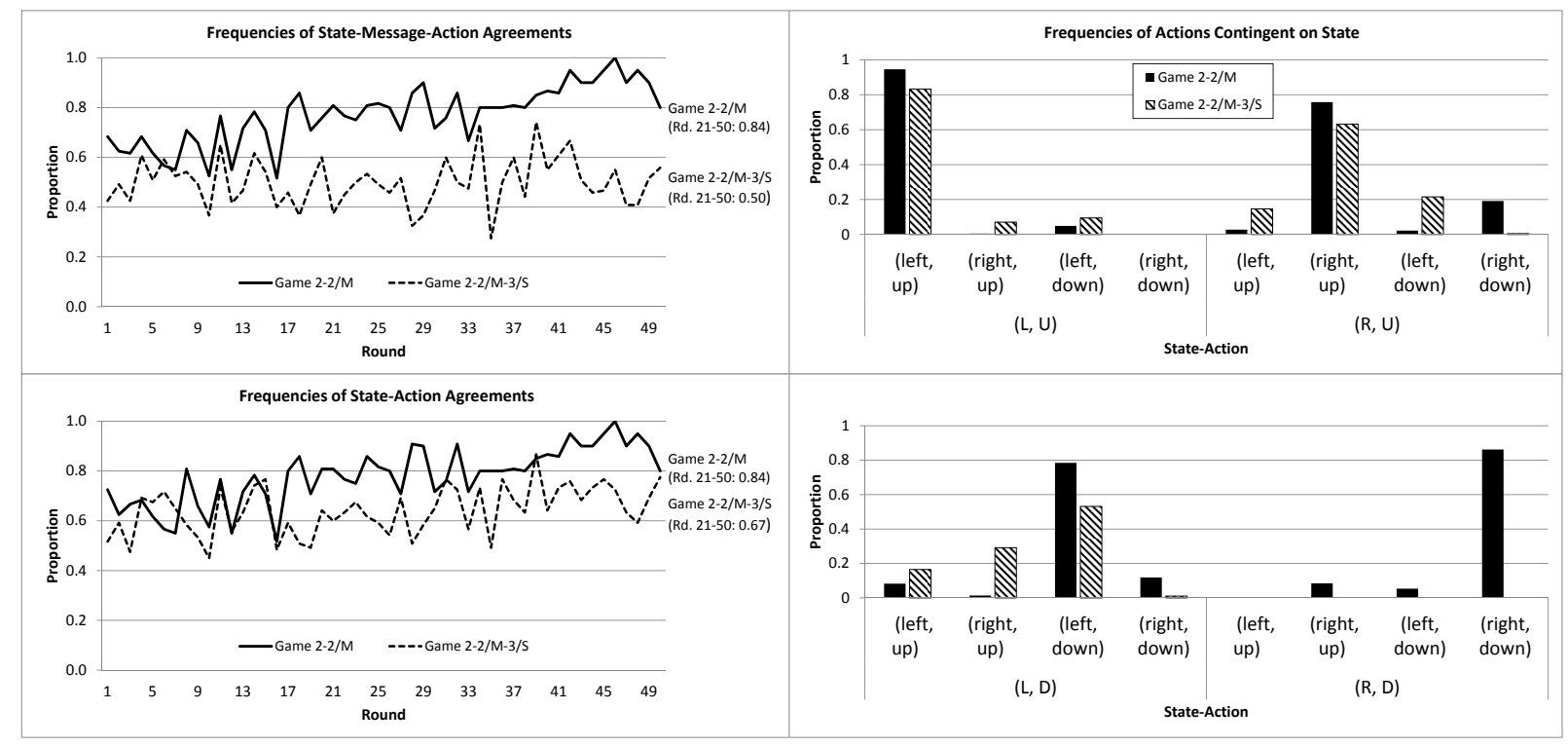

(a) Frequencies of State-(Message)-Action Agreements

(b) Frequencies of Actions Contingent on State

Figure 9: Information Revelation Outcomes in Games 2-2/M and 2-2/M-3/S

- Receivers in Game 2-2/M-3/S tended to follow the senders' recommendations less often, and their responses to irreconcilable messages justified the senders' deviations.

Even with binary messages, the type of senders' deviations observed in Game 2 resurfaced in Game 2-2/M-3/S. Figure 10 presents the senders' message uses and the receivers' responses in the two games. For Sender 1s in $(L, D)$, the frequency of "left" decreased from $89 \%$ in Game 2-2/M to $40 \%$ in Game 2-2/M-3/S; for Sender 2s in $(R, U)$, the frequency of "up" decreased from $79 \%$ to $41 \% .{ }^{41}$ Obtained under the tight control of what messages may be received in a given instance, the finding adds force to the idea that the uncertainty surrounding how receivers interpret messages that indicated inconsistency, in this case the irreconcilable ("right", "down"), was crucial to the senders' adherence. ${ }^{42}$ This finding also suggests that the high adherence observed in Game 2-2/M was a result of senders getting behind the veils of message frames and acting on incentives; when receivers were likely to respond to deviating messages with attractive actions, senders deviated despite the fact that the messages were framed according to the equilibrium-relevant dimensions.

\footnotetext{
${ }^{41}$ The decreases were statistically significant for the former $(p=0.0143$, Mann-Whitney test $)$ but not for the latter ( $p=0.1714$, Mann-Whitney test). The insignificance was accounted for by an outlier session in Game 2-2/M-3/S; the frequencies of "up" by Sender $2 \mathrm{~s}$ in $(R, U)$ were $20 \%, 25 \%, 30 \%$, and $90 \%$ in the four sessions.

${ }^{42}$ A sender's deviation was accurately anticipated by the other sender. Figure 14(b) in Appendix C presents the senders' predictions of the other senders' messages in Game 2-2/M-3/S as well as in Games 2-2/M and 2-LAB-2/M.
} 


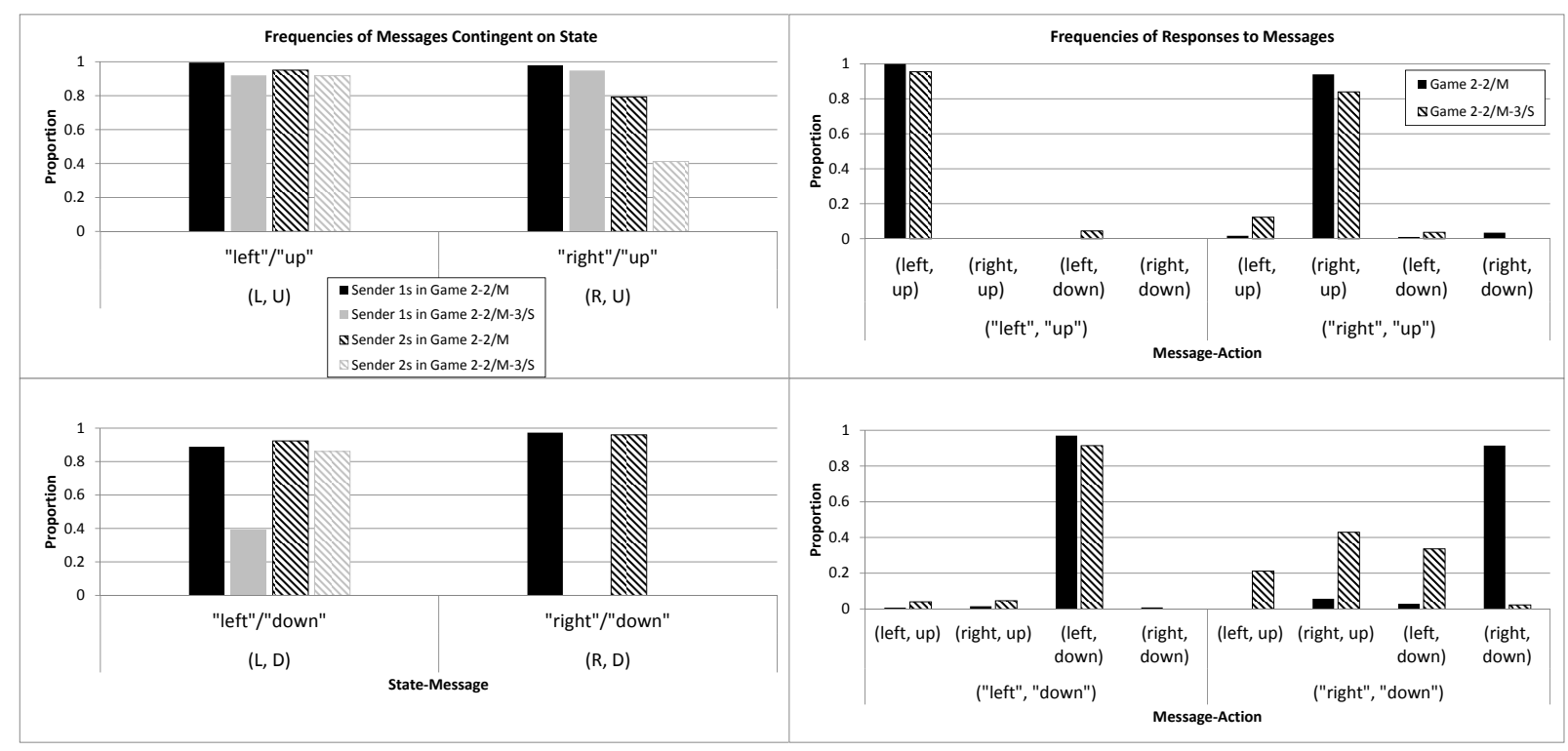

(a) Frequencies of Messages Contingent on State (b) Frequencies of Actions Contingent on Messages

Figure 10: Strategies in Games 2-2/M and 2-2/M-3/S

Although for message pairs ("left", "up"), ("right", "up"), and ("left", "down") receivers in Game 2-2/M-3/S still combined and followed the recommendations with high frequency, the deviations by senders did leave a noticeable trace on the receivers' responses: the frequencies in Game 2-2/M-3/S were 4\%-10\% lower than those in Game 2-2/M. Furthermore, the receivers' responses to the irreconcilable ("right", "down") indeed presented profitable opportunities for senders to deviate. The plausible, deviation-inviting responses, (right, up) and (left, down), were observed with frequencies $43 \%$ and $34 \%$. On the other hand, the implausible, deviation-deterring (left, up) was observed less often at $21 \%$, which is significantly lower than the threshold of $80 \%$ required to support the equilibrium $(p=0.0625$, Wilcoxon signed-rank test). The receivers' elicited beliefs further confirmed the implausibility of the supporting out-of-equilibrium beliefs: in the final-round predictions of the state, receivers in Game 2-2/M-3/S never predicted that the state was $(L, U)$ when ("right", "down") was received. ${ }^{43}$

The findings above address our last research question. The theoretical implausibility of the fully revealing equilibrium in Game 2-2/M-3/S (as supported by implausible out-ofequilibrium beliefs) translated into a lower adherence in the laboratory. Furthermore, the observed behavior and the elicited beliefs were consistent with the rationale behind why the equilibrium is implausible.

\footnotetext{
${ }^{43}$ Figure 15 in Appendix $\mathrm{C}$ presents the receivers' predictions in all games.
} 


\section{Concluding Remarks}

Battaglini (2002) provides a pioneering solution for how a decision maker in a multidimensional environment with misaligned interests can still extract full information through cheap talk by selectively listening to the experts on different issues. Using a series of simple games that capture the key logic of Battaglini's (2002) construction of fully revealing equilibria, we experimentally investigate this fundamental insight in the cheap-talk literature. We tackle the research task by asking three questions, which can be further subsumed into the following basic question: whether in the lab more information can be extracted with two senders via the strategies in Battaglini's (2002) equilibrium and what the deciding factors are.

Our findings confirm that, overall, receivers can extract more information in a multidimensional setting when there are two senders instead of one. The nature of dimensional common interests, the sizes of the message spaces, and the specification of out-ofequilibrium beliefs are all determinants of how much information can be transmitted. When payoffs are structured so that aligned interests exist on a dimension between a sender and the receiver in all states, irrespective of the other sender's behavior, truthful revelations as prescribed by the equilibrium are highly encouraged. Even when such aligned interests do not exist in cetain states, if the message spaces are restricted so that no inconsistent message can arise, substantial degree of equilibrium play is still observed. This points to the fact that the presence or absence of inconsistent messages (and how receivers respond to them) is crucial to the information transmission outcome. In this regard, restricting the message spaces, which eliminates these messages, facilitates equilibrium play. Nevertheless, if the equilibrium in question is supported by implausible out-of-equilibrium beliefs so that a plausible receiver's response invites deviation, information transmission is impeded even when the message spaces are restricted. This result substantiates the empirical importance of the robustness of an equilibrium, which is an important issue in the theoretical literature on multi-sender cheap talk.

We conclude by discussing three future research ideas that extend on our study. Similar to what we find, Blume et al. (2008) also document that restricting the message spaces expedites convergence to equilibrium, but the environment they consider is a single-sender game with a priori meaningless messages. To further explore the role of message spaces in multidimensional cheap talk, it would be interesting in our setting to implement meaningless messages or messages that do not match the action labels. We speculate that doing so would naturally reduce the extent of information transmission, but similar qualitative 
differences between two-dimensional and one-dimension messages may prevail.

In our inquiry regarding the effect of out-of-equilibrium belief specification, we explore how receivers would respond to a zero-probability event. While this is interesting from a theoretical perspective, in the real world we often face unlikely events rather than outright zero-probability events. An alternative design to address this more realistic situation in our setting would be to alter the prior probability distribution and assign a very small

probability to one state (instead of removing a state). The parallel question that may be addressed in future research would then be whether the receiver believes the senders if they recommend an action that is ideal for the unlikely state.

Our findings regarding message spaces suggest a policy implication for the design of institutions to elicit information: in a multidimensional environment with multiple experts, even when talk is otherwise cheap and cannot be verified, decision makers may still effectively elicit information if institutions are in place to restrict what experts can say and on what issues. Extrapolated from this observation is that decision makers' opportunity to commit to what to listen to and from whom, while theoretically having no impact in light of Battaglini's (2002) equilibrium, may have an effect in practice, and this presents another experimental inquiry that may be of interest.

As one of the first experimental studies on multidimensional cheap talk, we use a parsimonious design that allows us to identify, for example, the potentially policy-relevant effect of message spaces on information aggregation. Before experimentalists can "whisper in the ears of Princes" (Roth, 1995) with a more comprehensive picture on this and other relevant policy issues, additional experimental efforts will be needed, and we hope that our study will contribute to initiate further inquiries on this important topic, perhaps along the directions suggested above.

\section{References}

[1] Ambrus, A., and S. E. Lu (2014): "Almost Fully Revealing Cheap Talk with Imperfectly Informed Senders." Games and Economic Behavior, 88, 174-189.

[2] Ambrus, A., and S. Takahashi (2008): "Multi-Sender Cheap Talk with Restricted State Spaces." Theoretical Economics, 3, 1-27.

[3] Austen-Smith, D (1993): "Interested Experts and Policy Advice: Multiple Referrals under Open Rule." Games and Economic Behavior, 5, 3-43. 
[4] Battaglini, M (2002): "Multiple Referrals and Multidimensional Cheap Talk." Econometrica, 50, 1431-1451.

[5] Battaglini, M (2004): "Policy Advice with Imperfectly Informed Experts." Advances in Theoretical Economics, 4, Article 1.

[6] Battaglini, M., and U. Makarov (2011): "Cheap Talk with Multiple Audiences: An Experimental Analysis." Games and Economic Behavior, 83, 147-164.

[7] Binmore, K. (1999): "Why Experiment in Economics?" Economic Journal, 109, F16F24.

[8] Blume, A., D. V. DeJong, and G. B. Sprinkle (2008): "The Effect of Message Space Size on Learning and Outcomes in Sender-Receiver Games." Handbook of Experimental Economics Results, Elsevier.

[9] Blume, A., D. V. Dejong, Y.-G. Kim, and G. B. Sprinkle (1998): "Experimental Evidence on the Evolution of the Meaning of Messages in Sender-Recevier Games." American Economic Review, 88, 1323-1340.

[10] Blume, A., D. V. Dejong, Y.-G. Kim, and G. B. Sprinkle (2001): "Evolution of Communication with Partial Common Interest." Games and Economic Behavior, 37, 79120 .

[11] Blume, A., O. J. Board, and K. Kawamura (2007): "Noisy Talk." Theoretical Economics, 2, 395-440.

[12] Cai, H., and J. T.-Y. Wang (2006): "Overcommunication in Strategic Information Transmission Games." Games and Economic Behavior, 56, 7-36.

[13] Chakraborty, A., and R. Harbaugh (2007): "Comparative Cheap Talk." Journal of Economic Theory, 132, 70-94.

[14] Chakraborty, A., and R. Harbaugh (2010): "Persuasion by Cheap Talk." American Economic Review, 100: 2361-382.

[15] Crawford, V. P. (1998): "A Survey of Experiments on Communication via Cheap Talk." Journal of Economic Theory, 78, 286-298.

[16] Crawford, V. P. (2003): "Lying for Strategic Advantage: Rational and Boundedly Rational Mispresentation of Intentions." American Economic Review, 93, 133-149. 
[17] Crawford, V. P., and J. Sobel (1982): "Strategic Information Transmission." Econometrica, 50, 1431-1451.

[18] Dickhaut, J., K. McCabe, and A.t Mukherji (1995): "An Experimental Study of Strategic Information Transmission." Economic Theory, 6, 389-403.

[19] Farrell, J., and R. Gibbons (1989): "Cheap Talk with Two Audiences." American Economic Review, 79, 1214-1223.

[20] Fischbacher, U. (2007): "z-Tree: Zurich Toolbox for Ready-made Economic Experiments." Experimental Economics, 10, 171-178.

[21] Gilligan, T. W., and K. Krehbiel (1989): "Asymmetric Information and Legislative Rules with a Heterogeneous Committee." American Journal of Political Science, 33, 459-490.

[22] Gneezy, U. (2005): "Deception: The Role of Consequences." American Economic Review, 95, 384-394.

[23] Goltsman, M., J. H., G. Pavlov, and Francesco Squintani (2009): "Mediation, Arbitration and Negotiation." Journal of Economic Theory, 144, 1397-1420.

[24] Goltsman, M., and G. Pavlov (2011): "How to Talk to Multiple Audiences." Games and Economic Behavior, 72, 100-122.

[25] Ivanov, M. (2010): "Communication via a Strategic Mediator." Journal of Economic Theory, 145, 869-884.

[26] Jehiel, P. (2005): "Analogy-Based Expectation Equilibrium." Journal of Economic Theory, 123, 81-104.

[27] Jehiel, P., and F. Koessler (2008): "Revisiting Games of Incomplete Information with Analogy-Based Expectations." Games and Economic Behavior, 62, 533-557.

[28] Kawagoe, T., and H. Takizawa (2009): "Equilibrium Refinement vs. Level- $k$ Analysis: An Experimental Study of Cheap-Talk Games with Private Information." Games and Economic Behavior, 66, 238-255.

[29] Kim, C. (2010), "Non-robustness of Fully Revealing Equilibria in Cheap Talk Games." Mimeo, University of California, San Diego.

[30] Köszegi, B. (2006): "Emotional Agency." Quarterly Journal of Economics, 121, 121155. 
[31] Krishna, V., and J. Morgan (2001a): "A Model of Expertise." Quarterly Journal of Economics, 116, 747-775.

[32] Krishna, V., and J. Morgan (2001b): "Asymmetric Information and Legislative Rules: Some Amendments." American Political Science Review, 95, 435-452.

[33] Krishna, V., and J. Morgan (2004): "The Art of Conversation: Eliciting Information from Experts Through Multi-Stage Communication." Journal of Economic Theory, $117,147-179$.

[34] Levy, G., and R. Razin (2007): "On the Limits of Communication in Multidimensional Cheap Talk: A Comment." Econometrica, 75, 885-893.

[35] Lu, S. E. (2015): "Coordination-Free Equilibria in Cheap Talk Games." Mimeo.

[36] Minozzi, W., and J. Woon (2015): "Competition, Preference Uncertainty, and Jamming: A Strategic Communication Experiment." Mimeo.

[37] Morgan, J., and P. C. Stocken (2003): "An Analysis of Stock Recommendations." Rand Journal of Economics, 34, 183-203.

[38] Myerson, R. (1978): "Refinements of the Nash Equilibrium Concept." International Journal of Game Theory, 7, 73-80.

[39] Roth, A. E. (1995): "Introduction to Experimental Economics." in J. H. Kagel and A. E. Roth, eds., Handbook of Experimental Economics, Princteon, NJ: Princeton University Press, 3-109.

[40] Sánchez-Pagés, S., and M. Vorsatz (2007): "An Experimental Study of Truth-Telling in Sender-Receiver Game." Games and Economic Behavior, 61, 86-112.

[41] Sánchez-Pagés, S., and M. Vorsatz (2009): "Enjoy the Silence: An Experiment on Truth-Telling." Experimental Economics, 12, 220-241.

[42] Vespa, E., and A. J. Wilson (2014): "Communication With Multiple Senders: An Experiment." Mimeo.

[43] Wang, J. T.-Y., M. Spezio and, C. F. Camerer (2010): "Pinocchio's Pupil: Using Eyetracking and Pupil Dilation To Understand Truth Telling and Deception in SenderReceiver Games." American Economic Review, 100, 984-1007. 


\section{Appendix A - Preference Orders for Game 2-LAB (2- LAB-2/M) and Game 2-DAL}

Table 5: Preference Orders for Game 2-LAB (2-LAB-2/M)

\begin{tabular}{|c|c|c|c|c|}
\cline { 2 - 5 } \multicolumn{1}{c|}{} & $(L, U)$ & $(R, U)$ & $(L, D)$ & $(R, D)$ \\
\hline Sender 1 & $a_{R D}>a_{L U}>a_{L D}>a_{R U}$ & $a_{L D}>a_{R U}>a_{R D}>a_{L U}$ & $a_{R U}>a_{L D}>a_{L U}>a_{R D}$ & $a_{R U}>a_{R D}>a_{L U}>a_{L D}$ \\
\hline Sender 2 & $a_{R U}>a_{L U}>a_{L D}>a_{R D}$ & $a_{R D}>a_{R U}>a_{L U}>a_{L D}$ & $a_{R D}>a_{L D}>a_{L U}>a_{R U}$ & $a_{L D}>a_{R D}>a_{R U}>a_{L U}$ \\
\hline Receiver & $a_{L U}>a_{R U}>a_{R D}>a_{L D}$ & $a_{R U}>a_{L U}>a_{L D}>a_{R D}$ & $a_{L D}>a_{R U}>a_{R D}>a_{L U}$ & $a_{R D}>a_{L U}>a_{L D}>a_{R U}$ \\
\hline
\end{tabular}

Table 6: Preference Orders for Game 2-DAL

\begin{tabular}{|c|c|c|c|c|}
\cline { 2 - 5 } \multicolumn{1}{c|}{} & $(L, U)$ & $(R, U)$ & $(L, D)$ & $(R, D)$ \\
\hline Sender 1 & $a_{L D}>a_{L U}>a_{R D}>a_{R U}$ & $a_{R D}>a_{R U}>a_{L D}>a_{L U}$ & $a_{L U}>a_{L D}>a_{R U}>a_{R D}$ & $a_{R U}>a_{R D}>a_{L U}>a_{L D}$ \\
\hline Sender 2 & $a_{R U}>a_{L U}>a_{R D}>a_{L D}$ & $a_{L U}>a_{R U}>a_{L D}>a_{R D}$ & $a_{R D}>a_{L D}>a_{R U}>a_{L U}$ & $a_{L D}>a_{R D}>a_{L U}>a_{R U}$ \\
\hline Receiver & $a_{L U}>a_{R D}>a_{L D} \sim a_{R U}$ & $a_{R U}>a_{L D}>a_{L U} \sim a_{R D}$ & $a_{L D}>a_{R U}>a_{L U} \sim a_{R D}$ & $a_{R D}>a_{L U}>a_{L D} \sim a_{R U}$ \\
\hline
\end{tabular}

\section{Appendix B - Proofs}

Proof of Proposition 2. Game 1. Let $\mu=\left(\mu_{L U}, \mu_{R U}, \mu_{L D}, \mu_{R D}\right)$ be the receiver's beliefs, where $\mu_{H V}$ is the probability assigned to state $(H, V) \in\{L, R\} \times\{U, D\}$, and $U_{R}(a \mid \mu)$ be her expected payoff from action $a$ given beliefs $\mu$. We have that $U_{R}(($ left, up $) \mid \mu)=$ $50 \mu_{L U}+20 \mu_{R U}+20 \mu_{L D}, U_{R}(($ right, up $) \mid \mu)=20 \mu_{L U}+50 \mu_{R U}+20 \mu_{R D}, U_{R}(($ left, down $\left.) \mid \mu)\right)=$ $10 \mu_{L U}+50 \mu_{L D}+10 \mu_{R D}$, and $U_{R}($ (right, down $\left.) \mid \mu\right)=10 \mu_{R U}+10 \mu_{L D}+50 \mu_{R D}$. We first show that there exists a partially revealing equilibrium in which only $(L, U)$ is revealed so that the equilibrium information partition is $\{\{(L, U)\},\{(R, U),(L, D),(R, D)\}\}$. The receiver takes (left, up) when the state is $(L, U)$. In all other three states, the receiver's beliefs are $\mu_{R U}=\mu_{L D}=\mu_{R D}=\frac{1}{3}$ and $\mu_{L U}=0$. The receiver's best response is to randomize between (right, up) and (right, down) with probabilities $(p, 1-p), p \in[0,1]$. In order for the sender not to deviate, we require $20 \geqslant 10(1-p), 20 p+50(1-p) \geqslant 0$ and $50 p+20(1-p) \geqslant 10$ respectively in state $(L, U),(R, U)$ and $(R, D)$, which are satisfied for all $p \in[0,1]$. In 
$(L, D)$ we require $60 p \geqslant 15$, which is satisfied for any $p \geqslant \frac{1}{4}$. We show that other $1-3$ partitions are not consistent with equilibrium. Consider that only $(H, V) \neq(L, U)$ is fully revealed. The receiver takes $(h, v)$ when the state is $(H, V)$. In all other three states, the receiver's updated beliefs are $\mu_{\tilde{H} V}=\mu_{\tilde{H} \tilde{V}}=\mu_{H \tilde{V}}=\frac{1}{3}$ and $\mu_{H V}=0$ for $\tilde{H} \neq H, \tilde{V} \neq V$, and plays a best response. When $(H, V)=(R, U)$, the receiver's best response to the 3 -state partition is to randomize between (left, up) and (left, down) with probabilities $(p, 1-p)$, $p \in[0,1]$, so a sender in state $(L, D)$ has an incentive to tell that it is $(R, U)$ given that $60>15 p+20(1-p)$ for all $p \in[0,1]$. When $(H, V)=(L, D)$, the receiver's best response to the 3 -state partition is to take (right, up), so a sender in state $(L, D)$ has an incentive to tell that the state is in $\{(L, U),(R, U),(R, D)\}$ given that $60>20$. When $(H, V)=(R, D)$, the receiver's best response to the 3 -state partition is to take (left, up), so a sender in state $(R, U)$ has an incentive to tell that the state is $(R, D)$ given that $50>0$.

We next show that all $2-2$ partitions cannot constitute an equilibrium. First, consider a partially revealing equilibrium in which only dimension $H$ is revealed. When $H=L$ is revealed, the receiver's best response to the updated beliefs $\mu_{L U}=\mu_{L D}=\frac{1}{2}$ and $\mu_{R U}=$ $\mu_{R D}=0$ is to take (left, up). When $H=R$ is revealed, the receivers's best response to the updated beliefs $\mu_{R U}=\mu_{R D}=\frac{1}{2}$ and $\mu_{L U}=\mu_{L D}=0$ is (right, up). For a sender in $(L, D)$, equilibrium requires $15 \geqslant 60$, which is not satisfied. Next, consider a partially revealing equilibrium in which only dimension $V$ is revealed. When $V=U$ is revealed, the receiver's best response to the updated beliefs $\mu_{L U}=\mu_{R U}=\frac{1}{2}$ and $\mu_{L D}=\mu_{R D}=0$ is to randomize between (left, up) and (right, up) with probabilities $(p, 1-p)$ for any $p \in[0,1]$. When $V=D$ is revealed, the receiver's best response to the updated beliefs $\mu_{L U}=\mu_{R U}=0$ and $\mu_{L D}=\mu_{R D}=\frac{1}{2}$ is to randomize between (left, down) and (right, down) with probabilities $(q, 1-q)$ for any $q \in[0,1]$. For senders in states $(L, U)$ and $(R, U)$, equilibrium requires, respectively, $20 p \geqslant 50 q+10(1-q)$ and $20(1-p) \geqslant 10 q+50(1-q)$, which implies $20 \geqslant 60$, a contradiction. Finally, we show that the diagonal partition $\{\{(L, U),(R, D)\},\{(R, U),(L, D)\}\}$ is not consistent with equilibrium. When the main diagonal $\{(L, U),(R, D)\}$ is revealed, the receiver's best response to the updated beliefs $\mu_{L U}=\mu_{R D}=\frac{1}{2}$ and $\mu_{R U}=\mu_{L D}=0$ is to randomize between (left, up) and (right, down) with probabilities $(p, 1-p)$ for any $p \in[0,1]$. When the minor diagonal $\{(R, U),(L, D)\}$ is revealed, the receiver's best response to the updated beliefs $\mu_{L U}=\mu_{R D}=0$ and $\mu_{R U}=$ $\mu_{L D}=\frac{1}{2}$ is to randomize between (right, up) and (left, down) with probabilities $(q, 1-q)$ for any $q \in[0,1]$. For senders in states $(L, U)$ and $(R, D)$, equilibrium requires, respectively, $20 p+10(1-p) \geqslant 50(1-q)$ and $10 p+20(1-p) \geqslant 50 q$, which implies $30 \geqslant 50$, a contradiction.

We show that the fully revealing partition $\{\{(L, U)\},\{(L, D)\},\{(R, U)\},\{(R, D)\}\}$ can- 
not be sustained as equilibrium. It suffices to consider state $(L, U)$ in which the sender has an incentive to tell that it is $(L, D)$ given that he will receive 50 rather than 20 . To complete the proof, we rule out the $1-1-2$ partitions. There are six possible partitions here. The two partitions in which $V$ is fully revealed for fixed values of $H$ are also not feasible in equilibrium, because the sender shares no common interest with the receiver along dimension $V$. For each of the remaining four partitions, since when the state is one of the partially revealed ones the sender has an incentive to tell that it is one of the fully revealed ones (for this yields a payoff of 50 or 60), they also cannot be feasible in equilibrium.

Game 1-DAL. We have that $U_{R}(($ left, up $) \mid \mu)=50 \mu_{L U}+20 \mu_{R D}, U_{R}(($ right, up $) \mid \mu)=$ $50 \mu_{R U}+20 \mu_{L D}, U_{R}(($ left, down $\left.) \mid \mu)\right)=50 \mu_{L D}+20 \mu_{R U}$, and $U_{R}(($ right, down $) \mid \mu)=50 \mu_{R D}+$ $20 \mu_{L U}$. We first show the existence of the partially revealing equilibrium. Suppose the sender truthfully reveals $H=L$ and babbles on dimension $V$. The receiver's best response to her updated beliefs $\mu_{L U}=\mu_{L D}=\frac{1}{2}$ and $\mu_{R U}=\mu_{R D}=0$ (from the uniform prior) is to randomize between (left, up) and (left, down) with probabilities $(p, 1-p), p \in[0,1]$. Consider next that the sender truthfully reveals $H=R$ and babbles on dimension $V$. The receiver's best response to the updated beliefs $\mu_{R U}=\mu_{R D}=\frac{1}{2}$ and $\mu_{L U}=\mu_{L D}=0$ is to randomize between (right, up) and (right, down) with probabilities $(q, 1-q), q \in[0,1]$. In state $(L, U)$, we require that the sender has no incentive to tell that the state consists of $R$, or $20 p+50(1-p) \geqslant 10(1-q)$, which is satisfied for all $p \in[0,1]$ and all $q \in[0,1]$. Similarly, it is straightforward that for all $p \in[0,1]$ and all $q \in[0,1]$, the sender has no incentive to deviate in states $(R, U),(L, D)$ and $(R, D)$.

We show that there exists no equilibrium in Game 1-DAL with other information partitions. First, $\{\{(L, U)\},\{(L, D)\},\{(R, U)\},\{(R, D)\}\}$ cannot be sustained as equilibrium, for a sender in state $(L, U)$ would have an incentive to tell that it is $(L, D)$ given that he will receive 50 rather than 20 . Consider next the $1-3$ partition where only $(L, U)$ is fully revealed. In all other states, the receiver's best response to beliefs $\mu_{L D}=\mu_{R U}=\mu_{R D}=\frac{1}{3}$ and $\mu_{L U}=0$ is to randomize between (left, down) and (right, up) with probabilities $(p, 1-p), p \in[0,1]$. This does not constitute an equilibrium, because a sender in state $(L, D)$ has an incentive to tell that it is $(L, U)$ so the receiver takes (left, up), given that $50>20 p+10(1-p)$ for all $p \in[0,1]$. Similar arguments hold for all other 1-3 partitions.

We show next that other 2-2 partitions cannot constitute an equilibrium. Consider the partition where dimension $V$ is fully revealed. When $V=U$ is revealed, the receiver's best response to the updated beliefs $\mu_{L U}=\mu_{R U}=\frac{1}{2}$ and $\mu_{L D}=\mu_{R D}=0$ is to randomize between (left, up) and (right, up) with probabilities $(p, 1-p)$ for some $p \in[0,1]$. When $V=D$ is revealed, The receiver's best response to the updated beliefs $\mu_{L U}=\mu_{R U}=0$ and 
$\mu_{L D}=\mu_{R D}=\frac{1}{2}$ is to randomize between (left, down) and (right, down) with probabilities $(q, 1-q)$ for some $q \in[0,1]$. For senders in states $(L, U)$ and $(R, U)$, equilibrium requires, respectively, $20 p \geqslant 50 q+10(1-q)$ and $20(1-p) \geqslant 10 q+50(1-q)$, which implies $20 \geqslant 60$, a contradiction. For the partition $\{\{(L, U),(R, D)\},\{(L, D),(R, U)\}\}$ in which the diagonal is revealed, a similar argument shows that for senders in states $(L, U)$ and $(R, D)$, equilibrium requires, respectively, $50 q \leqslant 20 p+10(1-p)$ and $50(1-q) \leqslant 10 p+20(1-p)$, which leads to the contradiction of $50 \leqslant 30$.

We complete the proof by ruling out the six 1-1-2 partitions. By the same argument against the fully revealing partition, the two partitions in which $H$ is fully revealed for fixed values of $V$ cannot be sustained in equilibrium. The two other partitions in which $V$ is fully revealed for fixed values of $H$ are also not feasible in equilibrium, because the sender shares no common interest with the receiver along dimension $V$. This leaves partitions $\{\{(L, U),(R, D)\},\{(L, D)\},\{(R, U)\}\}$ and $\{\{(L, U)\},\{(R, D)\},\{(L, D),(R, U)\}\}$. However, senders in one of two partially revealed states have an incentive to tell that it is the fully revealed state that yields him a payoff of 50 .

Proof of Proposition 1. Game 2 and 2-DAL existence. We construct a fully revealing equilibrium in which Sender 1 truthfully reveals on dimension $H$ and Sender 2 on dimension $V$. To economize on notations, we denote $\left(h^{*}, v^{*}\right)$ to be the receiver's ideal action in state $(H, V) \in\{L, R\} \times\{U, D\}$. Consider the following senders' strategy profiles

$$
\sum_{\tilde{v} \in\{\text { up, down }\}} \sigma_{1}\left("\left(h^{*}, \tilde{v}\right) " \mid(H, V)\right)=1 \text {, and } \sum_{\tilde{h} \in\{\text { left, right }\}} \sigma_{2}\left("\left(\tilde{h}, v^{*}\right) " \mid(H, V)\right)=1 .
$$

for all $(H, V) \in\{L, R\} \times\{U, D\}$, in which Sender 1 truthfully reveals on dimension $H$ but is not required to truthfully reveal on dimension $V$ and Sender 2 does the exact opposite. The receiver's best responses are her ideal actions $\rho\left(\right.$ " $\left(h^{*}, v^{\prime}\right)$ ", " $\left(h^{\prime}, v^{*}\right)$ ") $)\left(h^{*}, v^{*}\right)$, because her updated beliefs (using Bayes' rule) are: For any $v^{\prime} \in\{$ up, down $\}$ and $h^{\prime} \in\{$ left, right $\}$,

$$
\begin{aligned}
\mu_{H V}\left(\left("\left(h^{*}, v^{\prime}\right) ", "\left(h^{\prime}, v^{*}\right) "\right)\right) & =\frac{\frac{1}{4} \sigma_{1}\left("\left(h^{*}, v^{\prime}\right) " \mid(H, V)\right) \sigma_{2}\left("\left(h^{\prime}, v^{*}\right) " \mid(H, V)\right)}{\sum_{(\tilde{H}, \tilde{V}) \in\{L, R\} \times\{U, D\}} \frac{1}{4} \sigma_{1}\left("\left(\tilde{h}^{*}, v^{\prime}\right) " \mid(\tilde{H}, \tilde{V})\right) \sigma_{2}\left("\left(h^{\prime}, \tilde{v}^{*}\right) " \mid(\tilde{H}, \tilde{V})\right)} \\
& =\frac{\sigma_{1}\left("\left(h^{*}, v^{\prime}\right) " \mid(H, V)\right) \sigma_{2}\left("\left(h^{\prime}, v^{*}\right) " \mid(H, V)\right)}{\sigma_{1}\left("\left(h^{*}, v^{\prime}\right) " \mid(H, V)\right) \sigma_{2}\left("\left(h^{\prime}, v^{*}\right) " \mid(H, V)\right)}=1,
\end{aligned}
$$


since either $\sigma_{1}\left("\left(h^{*}, v^{\prime}\right) " \mid(\tilde{H}, \tilde{V})\right)=0$ or $\sigma_{2}\left("\left(h^{\prime}, v^{*}\right) " \mid(\tilde{H}, \tilde{V})\right)=0$ unless $(\tilde{H}, \tilde{V})=(H, V)$.

To verify that (B.1) constitutes an equilibrium, note that given the strategies of Sender 2 and the receiver, Sender 1 can only influence the receiver in the choice between $\left(h^{*}, v^{*}\right)$ and $\left(\tilde{h}, v^{*}\right), h^{*} \neq \tilde{h}$; it is straightforward that Sender 1 strictly prefers $\left(h^{*}, v^{*}\right)$ over $\left(\tilde{h}, v^{*}\right)$. Similarly, Sender 2 can only influence the receiver in the choice between $\left(h^{*}, v^{*}\right)$ and $\left(h^{*}, \tilde{v}\right)$ where he strictly prefers $\left(h^{*}, v^{*}\right)$ over $\left(h^{*}, \tilde{v}\right)$. Other than (B.1), there is no restriction on $\sigma_{1}$ ("( $h^{*}$, up)" $\left.\mid(H, V)\right), \sigma_{1}$ ("( $h^{*}$, down)" $\left.\mid(H, V)\right), \sigma_{2}$ ("(left, $\left.\left.v^{*}\right) " \mid(H, V)\right)$ and $\sigma_{2}$ ("(right, $\left.v^{*}\right)$ ") $\left.(H, V)\right)$. If $\sigma_{1}\left("\left(h^{*}, v^{*}\right)\right.$ ") $\left.\mid(H, V)\right)=\sigma_{2}\left("\left(h^{*}, v^{*}\right)\right.$ " $\left.\mid(H, V)\right)=1$, we obtain the third class of equilibrium. The receiver's response after receiving an out-of-equilibrium inconsistent message pair can be assigned to be one of the equilibrium responses, which suffice to deter deviations. If $\sigma_{1}$ ("( $h^{*}$, up $\left.) " \mid(H, V)\right)>0, \sigma_{1}$ ("( $h^{*}$, down)" $\left.\mid(H, V)\right)>0$, $\sigma_{2}$ ("(left, $\left.\left.v^{*}\right) " \mid(H, V)\right)>0$ and $\sigma_{2}$ ("(right, $\left.\left.v^{*}\right) " \mid(H, V)\right)>0$, we obtain the first class of equilibrium, in which there is no out-of-equilibrium message pair.

Game 2 non-diagonal. Here we prove the non-existence of diagonal fully revealing equilibria for Game 2. If Sender 1 reveals partition $\{\{(L, U),(R, D)\},\{(R, U),(L, D)\}\}$, Sender 2 in state $(R, U)$ has an incentive to tell that the state consists of $D$ to induce action (left, down). If Sender 2 reveals partition $\{\{(L, U),(R, D)\},\{(R, U),(L, D)\}\}$, Sender 1 in state $(L, D)$ has an incentive to tell that the state consists of $U$ to induce (right, up).

Game 2-LAB existence \& non-diagonal. Omitted as it is a relabeling of Game 2.

Game 2-DAL diagonal. For the second class of fully revealing equilibrium in which Sender 1 reveals between diagonals and Sender 2 reveals on dimension $V$, the receiver's best response (to the updated beliefs) is to take her ideal action $\rho\left("\left(h^{*}, v^{*}\right)\right.$ or $(\tilde{h}, \tilde{v})$ ", " $\left(h^{\prime}, v^{*}\right)$ ") $=$ $\left(h^{*}, v^{*}\right)$ for $h^{*} \neq \tilde{h}$ and $v^{*} \neq \tilde{v}$. Given the strategies of Sender 1 and the receiver, Sender 2 can only influence the receiver in the choice between $\left(h^{*}, v^{*}\right)$ and $(\tilde{h}, \tilde{v})$, but he strictly prefers $\left(h^{*}, v^{*}\right)$ over $(\tilde{h}, \tilde{v})$. Similarly, Sender 1 , given the others' strategies, can only influence the receiver in the choice between $\left(h^{*}, v^{*}\right)$ and $\left(\tilde{h}, v^{*}\right)$ where he strictly prefers $\left(h^{*}, v^{*}\right)$ over $\left(\tilde{h}, v^{*}\right)$. For the last class of fully revealing equilibrium in which Sender 1 reveals on dimension $H$ and Sender 2 reveals between diagonals, the receiver's best response (to the updated beliefs) is to take her ideal action $\rho\left("\left(h^{*}, v^{\prime}\right)\right.$ ", " $\left(h^{*}, v^{*}\right)$ or $(\tilde{h}, \tilde{v})$ ") $=\left(h^{*}, v^{*}\right)$ for $h^{*} \neq \tilde{h}$ and $v^{*} \neq \tilde{v}$. Given the strategies of Sender 1 and the receiver, Sender 2 can only influence the receiver in the choice between $\left(h^{*}, v^{*}\right)$ and $\left(h^{*}, \tilde{v}\right), v^{*} \neq \tilde{v}$, but he strictly prefers $\left(h^{*}, v^{*}\right)$ over $\left(h^{*}, \tilde{v}\right)$. Similarly, Sender 1 , given the others' strategies, can only influence the receiver in the choice between $\left(h^{*}, v^{*}\right)$ and $(\tilde{h}, \tilde{v}), h^{*} \neq \tilde{h}$ and $v^{*} \neq \tilde{v}$, where he strictly prefers $\left(h^{*}, v^{*}\right)$ over $(\tilde{h}, \tilde{v})$. 
Proof of Proposition 3. With the binary message spaces the senders' strategy profiles in (B.1) become $\sigma_{1}($ " $h " \mid(H, V))=\sigma_{2}(" v " \mid(H, V))=1$. The receiver updates her beliefs in a similar fashion as in (B.2), and her best response is $\rho($ " $h "$, " $v ")=(h, v)$. Similar to the argument in the proof of Proposition 1, the senders' strategies also constitute best responses. There are two other classes of strategy profiles to achieve full revelation: 1) Sender 1 truthfully revealing on dimension $V$ and Sender 2 on dimension $H$, and 2) one sender truthfuly reveals on the diagonal, and the other sender reveals on either dimension $V$ or dimension $H$. It is straightforward to verify that neither of these strategy profile can constitute an equilibrium. Given that under the binary message spaces there is no out-of-equilibrium message pair for any fully revealing equilibrium, the receiver's beliefs are always derived from Bayes' rule.

Proof of Proposition 4. Consider $\sigma_{1}(" h " \mid(H, V))=\sigma_{2}(" v " \mid(H, V))=1$, where the receiver's best response is $\rho(" h ", " v ")=(h, v)$. It is straightforward that in state $(L, U)$ no sender has an incentive to deviate, so we specify the receiver's response to an irreconcilable message pair to ensure non-deviation in states $(R, U)$ and $(L, D)$. Given $\mu=$ $\left(\mu_{L U}, \mu_{L D}, \mu_{R U}\right)$, the receiver's expected payoffs are $U_{R}(($ left, up $) \mid \mu)=50 \mu_{L U}+20\left(\mu_{R U}+\right.$ $\left.\mu_{L D}\right), U_{R}(($ right, up $) \mid \mu)=20 \mu_{L U}+50 \mu_{R U}, U_{R}(($ left, down $\left.) \mid \mu)\right)=10 \mu_{L U}+50 \mu_{L D}$, and $U_{R}(($ right, down $) \mid \mu)=10\left(\mu_{R U}+\mu_{L D}\right)$. For any $\mu, U_{R}(($ right, down $) \mid \mu)<U_{R}(($ left, up $) \mid \mu)$. Thus, (right, down) is strictly dominated. Let the receiver take (left, up), (right, up) and (left, down) with respective probabilities $p, q$ and $1-p-q$ after an irreconcilable message pair. Then, Sender 1 in state $(L, D)$ will have no incentive to tell that the state consists of $R$ only if $20 \geqslant 15 p+60 q+20(1-p-q)$ or $p \geqslant 8 q$. Sender 2 in state $(R, U)$ will have no incentive to tell that the state consists of $D$ only if $20 \geqslant 15 p+20 q+60(1-p-q)$ or $9 p+8 q \geqslant 8$. Combining $p \geqslant 8 q$ and $9 p+8 q \geqslant 8$, we obtain $p \geqslant \frac{4}{5}$ as required. Similar to Game 2-2/M, other classes of strategy profiles cannot constitute an equilibrium so that $\sigma_{1}($ " $h$ " $\mid(H, V))=\sigma_{2}($ "v" $\mid(H, V))=1$ represent the unique strategy profiles that does.

Proof of Corollary 1. To support the fully revealing equilibrium, the receiver's strategy after an irreconcilable message pair needs to put probability of at least $\frac{4}{5}$ on (left, up), so the out-of-equilibrium beliefs have to assign positive probability on $(L, U)$. In an $\varepsilon$-perturbed 
game, after an irreconcilable message pair the receiver's belief that the state is $(L, U)$ is

$$
\mu_{L U}\left(\sigma, g, \varepsilon^{n}\right)=\frac{\frac{1}{3} \varepsilon_{1}^{n} g_{1}^{R U} \varepsilon_{2}^{n} g_{2}^{L D}}{\frac{1}{3} \varepsilon_{1}^{n} g_{1}^{R U} \varepsilon_{2}^{n} g_{2}^{L D}+\frac{1}{3} \varepsilon_{2}^{n} g_{2}^{L D}+\frac{1}{3} \varepsilon_{1}^{n} g_{1}^{R U}},
$$

where $g_{i}^{H V}$ is the probability that Sender $i$ observes state $(H, V)$ in the event of mistake. For $g_{1}^{R U}>0$ and $g_{2}^{L D}>0, \mu_{L U}\left(\sigma, g, \varepsilon^{n}\right) \rightarrow 0$ as $\varepsilon^{n} \rightarrow 0$ for any $\varepsilon^{n}$ converging to zero. Hence, there exists no $g=\left(g_{1}, g_{2}\right)$ so that the beliefs induced by equilibrium strategies $\sigma=\left(\sigma_{1}, \sigma_{2}\right)$ in an $\varepsilon$-perturbed game put positive probability on $(L, U)$ as $\varepsilon^{n} \rightarrow 0$.

Proof of Corollary 2. Since the first two class of fully revealing equilibria are free of outof-equilibrium beliefs, they are robust. We provide an example of non-robust (third class) equilibrium in which both sender send " $(h, v)$ " for state $(H, V)$. It suffices to consider one inconsistent message pair. Let the equilibrium be supported by out-of-equilibrium beliefs that assign probability one to $(L, U)$ after ("(right, down)", "(right, up)"); the receiver takes action (left, up) to deter deviations by Sender 1 in state $(R, U)$ and Sender 2 in state $(R, D)$. Upon receiving ("(right, down)", "(right, up)") in the corresponding equilibrium in an $\varepsilon$-perturbed game, the receiver's beliefs that the state is $(L, U)$ is

$$
\mu_{L U}\left(\sigma, g, \varepsilon^{n}\right)=\frac{\frac{1}{4} \varepsilon_{1}^{n} g_{1}^{R D} \varepsilon_{2}^{n} g_{2}^{R U}}{\frac{1}{4} \varepsilon_{1}^{n} g_{1}^{R D} \varepsilon_{2}^{n} g_{2}^{R U}+\frac{1}{4} \varepsilon_{1}^{n} g_{1}^{R D}+\frac{1}{4} \varepsilon_{1}^{n} g_{1}^{R D} \varepsilon_{2}^{n} g_{2}^{R U}+\frac{1}{4} \varepsilon_{2}^{n} g_{2}^{R U}}
$$

For $g_{1}^{R D}>0$ and $g_{2}^{R U}>0, \mu_{L U}\left(\sigma, g, \varepsilon^{n}\right) \rightarrow 0$ as $\varepsilon^{n} \rightarrow 0$ for any $\varepsilon^{n}$ converging to zero.

Proof of Corollary 3. All fully revealing equilibria are free of out-of-equilibrium beliefs. 


\section{Appendix C - Tables and Figures}

Table 7: Summary Statistics

\begin{tabular}{|c|c|c|c|c|c|c|c|c|c|c|}
\hline & & Rounds & $\begin{array}{c}(1) \\
\text { Game } \\
1\end{array}$ & $\begin{array}{c}(2) \\
\text { Game } \\
1-D A L\end{array}$ & $\begin{array}{c}(3) \\
\text { Game } \\
2\end{array}$ & $\begin{array}{c}(4) \\
\text { Game } \\
2-D A L\end{array}$ & $\begin{array}{c}(5) \\
\text { Game } \\
2-2 / \mathrm{M}\end{array}$ & $\begin{array}{c}(6) \\
\text { Game } \\
2-2 / \mathrm{M}-3 / \mathrm{S}\end{array}$ & $\begin{array}{c}(7) \\
\text { Game } \\
2-\mathrm{LAB}\end{array}$ & $\begin{array}{c}(8) \\
\text { Game } \\
\text { 2-LAB-2/M }\end{array}$ \\
\hline \multicolumn{11}{|c|}{ A. Frequencies of State-Message Agreements } \\
\hline \multirow{3}{*}{ (1) } & Sender 1 & $1-20$ & 0.76 & 0.95 & 0.72 & 0.87 & 0.88 & 0.77 & 0.61 & - \\
\hline & State-Message & $21-50$ & 0.69 & 0.99 & 0.77 & 0.89 & 0.96 & 0.77 & 0.60 & - \\
\hline & $(H,.) \rightleftarrows$ “ $(h,) "$. & $41-50$ & 0.69 & 0.99 & 0.83 & 0.93 & 0.99 & 0.77 & 0.55 & - \\
\hline \multirow{3}{*}{ (2) } & Sender 1 & $1-20$ & 0.48 & 0.45 & 0.53 & 0.49 & - & - & 0.49 & - \\
\hline & State-Message & $21-50$ & 0.58 & 0.50 & 0.50 & 0.39 & - & - & 0.50 & - \\
\hline & $(., V) \rightleftarrows "(., v) "$ & $41-50$ & 0.62 & 0.44 & 0.51 & 0.35 & - & - & 0.43 & - \\
\hline \multirow{3}{*}{ (3) } & Sender 1 & $1-20$ & $\begin{array}{lll}- & & \end{array}$ & - & 0.52 & 0.53 & - & - & 0.68 & 0.88 \\
\hline & State-Message & $21-50$ & - & - & 0.59 & 0.44 & - & - & 0.72 & 0.92 \\
\hline & $(H, V) \backslash \searrow "(h, v) "$ & $41-50$ & - & - & 0.62 & 0.38 & $\begin{array}{cccc}- \\
-\end{array}$ & - & 0.70 & 0.94 \\
\hline \multirow{3}{*}{ (4) } & Sender 2 & $1-20$ & - & - & 0.61 & 0.45 & - & - & 0.56 & - \\
\hline & State-Message & $21-50$ & - & - & 0.51 & 0.47 & - & - & 0.60 & - \\
\hline & $(H,.) \rightleftarrows "(h,) "$. & $41-50$ & - & - & 0.47 & 0.50 & - & - & 0.62 & - \\
\hline \multirow{3}{*}{ (5) } & Sender 2 & $1-20$ & - & - & 0.78 & 0.89 & 0.83 & 0.75 & 0.78 & 0.83 \\
\hline & State-Message & $21-50$ & - & - & 0.75 & 0.94 & 0.91 & 0.72 & 0.73 & 0.94 \\
\hline & $(., V) \rightleftarrows "(., v) "$ & $41-50$ & - &  & 0.75 & 0.93 & 0.94 & 0.73 & 0.74 & 0.96 \\
\hline \multicolumn{11}{|c|}{ B. Frequencies of Message-Action Agreements } \\
\hline \multirow{3}{*}{ (1) } & Sender 1 & $1-20$ & 0.85 & 0.86 & 0.75 & 0.85 & 0.96 & 0.77 & 0.61 & - \\
\hline & Message-Action & $21-50$ & 0.79 & 0.89 & 0.82 & 0.91 & 0.98 & 0.78 & 0.56 & - \\
\hline & $(H,.) \rightleftarrows$ " $(h,) "$. & $41-50$ & 0.78 & 0.92 & 0.83 & 0.93 & 0.99 & 0.77 & 0.54 & - \\
\hline \multirow{3}{*}{ (2) } & Sender 1 & $1-20$ & 0.45 & 0.58 & 0.47 & 0.51 & - & - & 0.47 & - \\
\hline & Message-Action & $21-50$ & 0.48 & 0.54 & 0.50 & 0.43 & - & - & 0.52 & - \\
\hline & $(., V) \rightleftarrows "(., v) "$ & $41-50$ & 0.48 & 0.62 & 0.51 & 0.42 & - & - & 0.49 & - \\
\hline \multirow{3}{*}{ (3) } & Sender 1 & $1-20$ & - & - & 0.53 & 0.53 & - & - & 0.76 & 0.90 \\
\hline & Message-Action & $21-50$ & - & - & 0.56 & 0.47 & - & - & 0.80 & 0.97 \\
\hline & $(H, V) \backslash \searrow "(h, v) "$ & $41-50$ & - & - & 0.58 & 0.45 & - & - & 0.83 & 0.99 \\
\hline \multirow{3}{*}{ (4) } & Sender 2 & $1-20$ & - & - & 0.48 & 0.50 & - & - & 0.57 & - \\
\hline & Message-Action & $21-50$ & - & - & 0.48 & 0.48 & - & - & 0.62 & - \\
\hline & $(H,.) \rightleftarrows "(h,) "$. & $41-50$ & - & - & 0.46 & 0.48 & - & - & 0.62 & - \\
\hline \multirow{3}{*}{ (5) } & Sender 2 & $1-20$ & - & - & 0.76 & 0.89 & 0.94 & 0.75 & 0.75 & 0.93 \\
\hline & Message-Action & $21-50$ & - & - & 0.78 & 0.92 & 0.97 & 0.74 & 0.80 & 0.99 \\
\hline & $(., V) \rightleftarrows$ " $(., v) "$ & $41-50$ & - & - & 0.83 & 0.92 & 0.98 & 0.74 & 0.78 & 1.00 \\
\hline \multicolumn{11}{|c|}{ C. Frequencies of State-Action/State-Message-Action Agreements } \\
\hline \multirow{3}{*}{ (1) } & State-Action & $1-20$ & 0.71 & 0.82 & 0.63 & 0.76 & 0.85 & 0.71 & 0.58 & 0.69 \\
\hline & $(H,.) \rightleftarrows(h,)$. & $21-50$ & 0.64 & 0.89 & 0.69 & 0.83 & 0.94 & 0.75 & 0.61 & 0.84 \\
\hline & & $41-50$ & 0.64 & 0.91 & 0.76 & 0.88 & 0.98 & 0.78 & 0.62 & 0.89 \\
\hline \multirow{3}{*}{ (2) } & State-Action & $1-20$ & 0.55 & 0.51 & 0.68 & 0.81 & 0.79 & 0.72 & 0.67 & 0.77 \\
\hline & $(., V) \rightleftarrows(., v)$ & $21-50$ & 0.56 & 0.50 & 0.66 & 0.86 & 0.89 & 0.75 & 0.64 & 0.93 \\
\hline & & $41-50$ & 0.51 & 0.50 & 0.72 & 0.87 & 0.92 & 0.77 & 0.65 & 0.96 \\
\hline \multirow{3}{*}{ (3) } & State-Action & $1-20$ & 0.39 & 0.42 & 0.44 & 0.62 & 0.68 & 0.60 & 0.45 & 0.65 \\
\hline & $(H, V) \rightleftarrows(h, v)$ & $21-50$ & 0.39 & 0.45 & 0.48 & 0.73 & 0.84 & 0.67 & 0.46 & 0.84 \\
\hline & & $41-50$ & 0.38 & 0.47 & 0.58 & 0.77 & 0.91 & 0.71 & 0.46 & 0.89 \\
\hline & tate-Message-Action & $1-20$ & - & - & 0.35 & 0.59 & 0.67 & 0.50 & - & - \\
\hline & $(H, V) \widetilde{\sim}(h, v)$ & $21-50$ & - & - & 0.39 & 0.71 & 0.84 & 0.50 & - & - \\
\hline & $("(h,$.$) ", "(.,v)")$ & $41-50$ & - & - & 0.47 & 0.76 & 0.91 & 0.52 & - & - \\
\hline
\end{tabular}

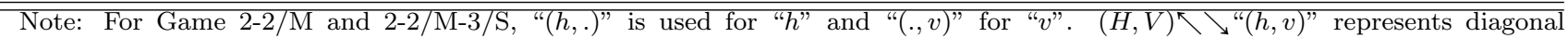
agreements. The numbers in bold indicate the (major) equilibrium-relevant dimensions. 


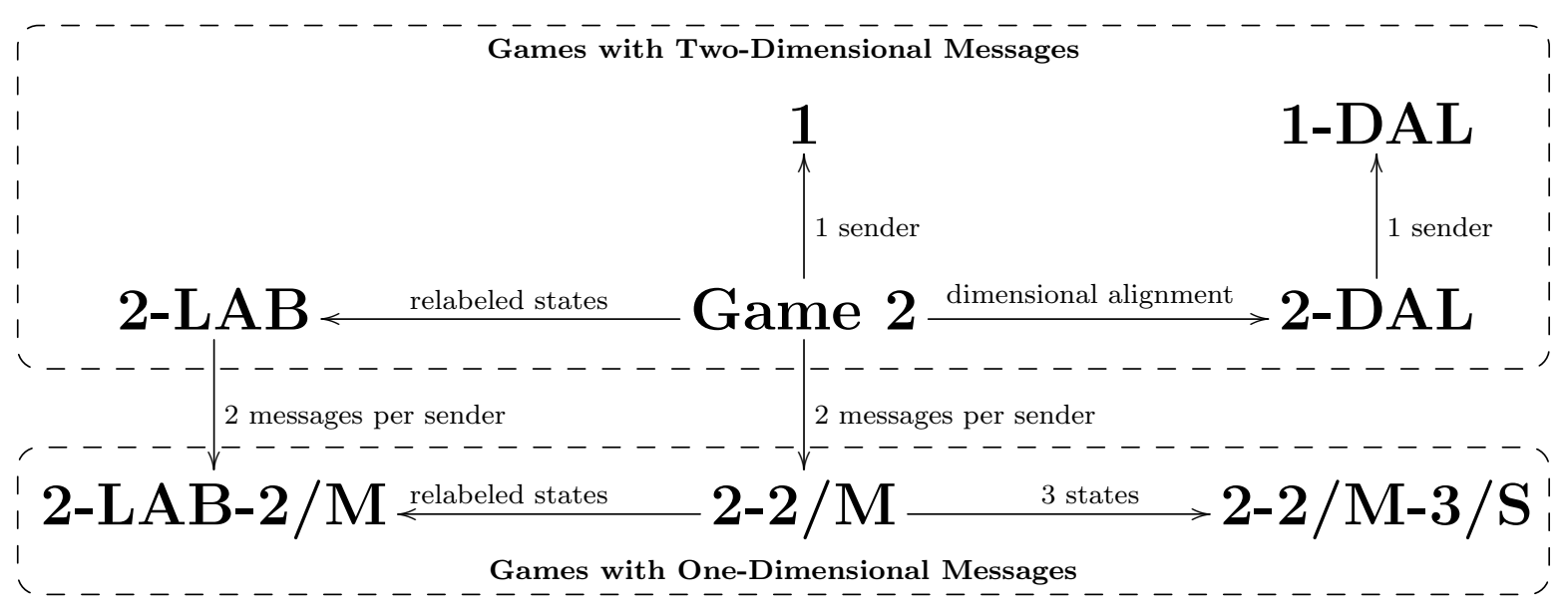

Figure 11: Experimental Design



Figure 12: Frequencies of State-Action Agreements: Games 2-DAL and 1-DAL 


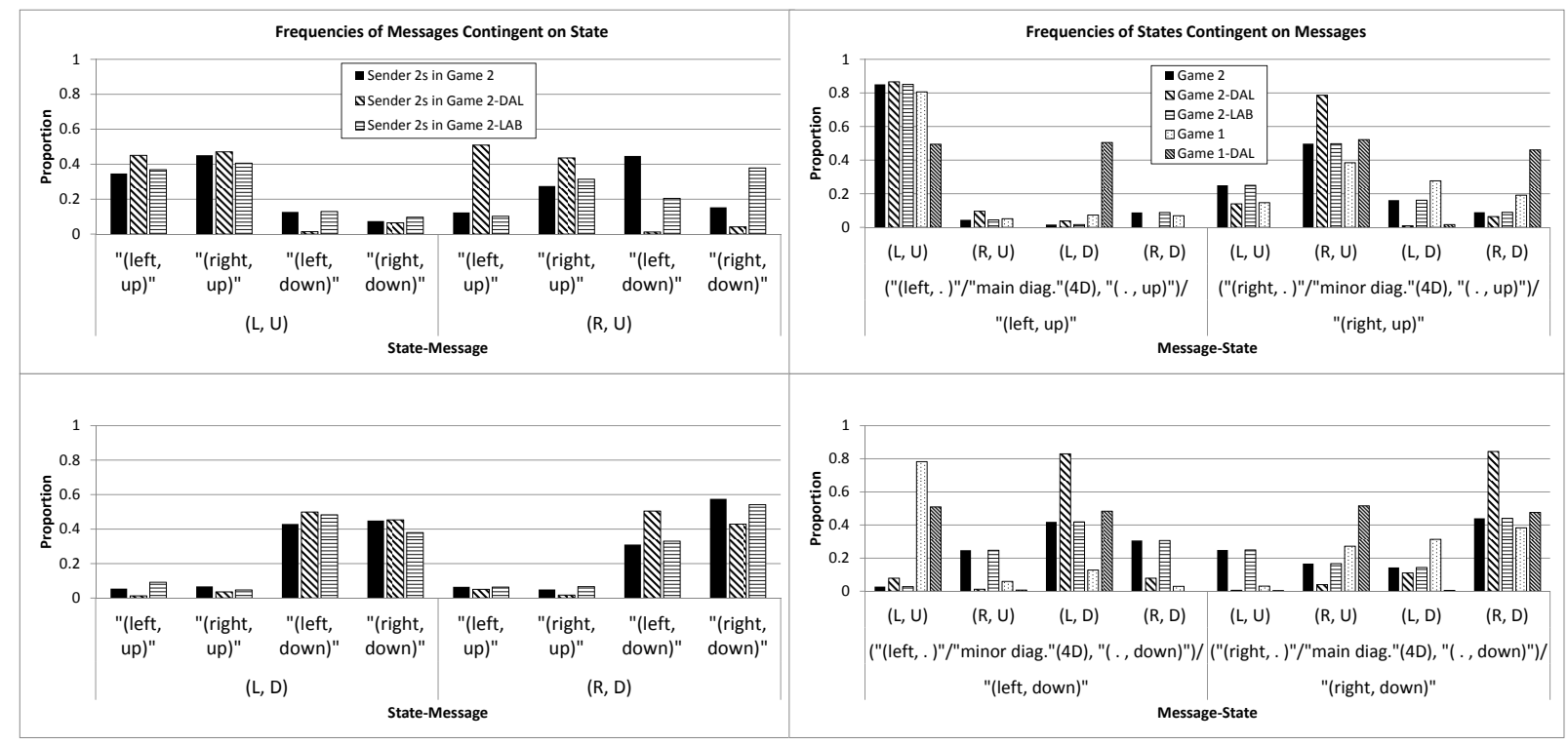

(a) Frequencies of Messages Contingent on State (Sender $2 \mathrm{~s}$ )

(b) Frequencies of States Contingent on Message Pairs

Figure 13: Sender 2s' Strategies in Games 2, 2-DAL, and 2-LAB, and Conditional Distributions of States Implied by Message Uses in All Two-Dimensional Message Games

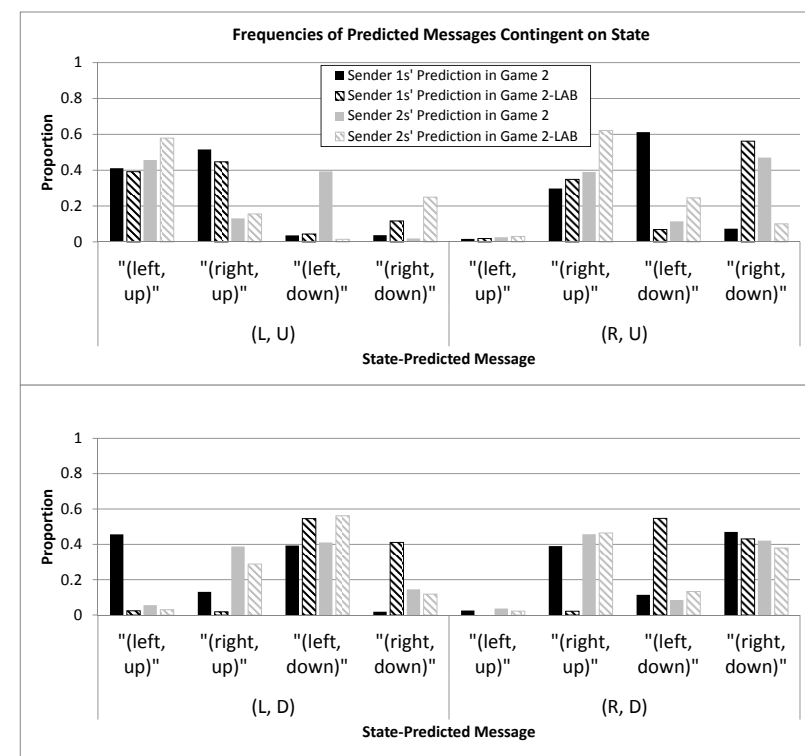

(a) Frequencies of Predicted Messages Contingent on State: Games 2 and 2-LAB

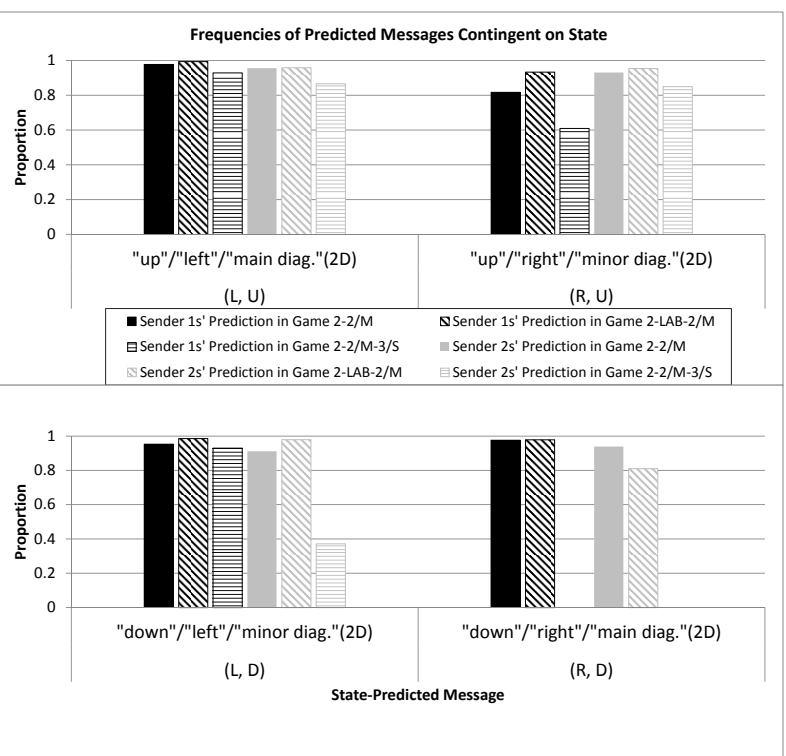

(b) Frequencies of Predicted Messages Contingent on State: Games 2-2/M, 2-LAB-2/M, and

$$
2-2 / \mathrm{M}-3 / \mathrm{S}
$$

Figure 14: Senders' Predictions of the Other Sender's Messages 


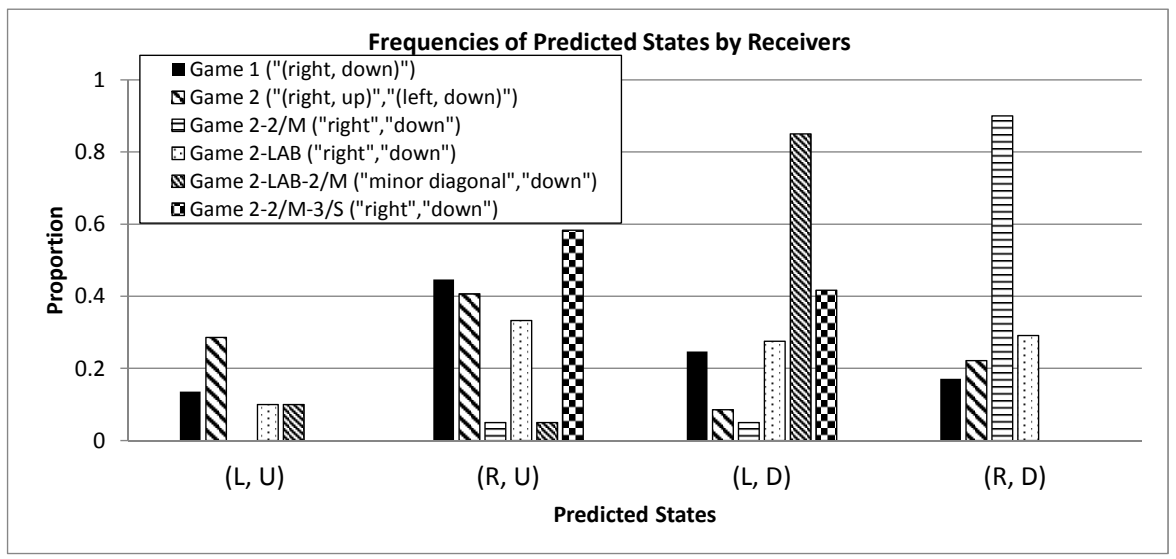

Figure 15: Receivers' Predictions of State Contingent on Messages 